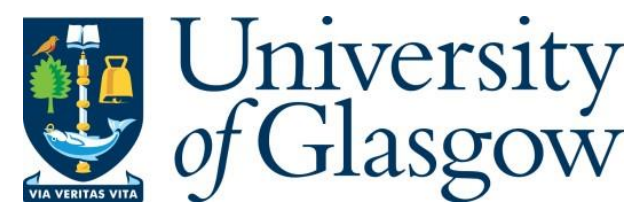

Cimbri, D., Wang, J., Al-Khalidi, A. and Wasige, E. (2022) Resonant tunnelling diodes high-speed terahertz wireless communications - a review. IEEE Transactions on Terahertz Science and Technology, (doi: 10.1109/TTHZ.2022.3142965).

There may be differences between this version and the published version. You are advised to consult the publisher's version if you wish to cite from it.

https://eprints.gla.ac.uk/263093/

Deposited on: 13 January 2022

Enlighten - Research publications by members of the University of Glasgow https://eprints.gla.ac.uk 


\title{
Resonant Tunnelling Diodes High-Speed Terahertz Wireless Communications - A Review
}

\author{
Davide Cimbri ${ }^{\circledR}$, Jue Wang, Abdullah Al-Khalidi ${ }^{\circledR}$, Senior Member, IEEE, and Edward Wasige ${ }^{\circledR}$, Member, IEEE
}

\begin{abstract}
Resonant tunnelling diode (RTD) technology is emerging as one of the promising semiconductor-based solidstate technologies for terahertz (THz) wireless communications. This paper provides a review of the state-of-the-art, with a focus on the THz RTD oscillator, which is the key component of RTDbased THz transmitters and coherent receivers. A brief summary on the device principle of operation, technology, modelling, as well as an overview of oscillator design and implementation approaches for $\mathrm{THz}$ emitters, is provided. A new insight to device evaluation and to the reported oscillator performance levels is also given, together with brief remarks on RTD-based THz detectors. Thereafter, an overview of the reported wireless links which utilise an RTD in either transmission or reception, or in both roles, is given. Highlight results include the record single-channel wireless data rate of $56 \mathrm{~Gb} / \mathrm{s}$ employing an all RTD-based transceiver, which demonstrates the potential of the technology for future short-range communications. The paper concludes with a discussion of the current technical challenges and possible strategies for future progress.
\end{abstract}

Index Terms-Terahertz, wireless communications, resonant tunnelling diode, electronic oscillator, terahertz monolithic integrated circuit.

\section{INTRODUCTION}

$\mathbf{F}$ OR the last two decades, we have witnessed an extraordinarily fast evolution of mobile cellular networks, starting from first generation $(1 \mathrm{G})$ to fourth generation $(4 \mathrm{G})$, and with the fifth generation $(5 \mathrm{G})$ of wireless communication networks now being deployed [1]. Indeed, the tremendous increase in mobile data traffic and wireless networks widespread diffusion is facing the unceasing demand for ultra-broadband multigigabit wireless communication technology, capable of extremely large channel bandwidths and ultra-high data rates required by modern multimedia services [2], including the Internet of Things [3]. This is in line with Edholm's law [4], which states that the demand for bandwidth performance in wireless short-range communications has doubled every 18 months since 1980 [5], and so data rates of tens of gigabits per second $(\mathrm{Gb} / \mathrm{s})$ [6] had to be accommodated since around 2020 onwards [7], while hundreds of Gb/s [8] and even

Manuscript received month day, year; revised month day, year; accepted month day, year. The work of Davide Cimbri was supported by TeraApps (Doctoral Training Network in Terahertz Technologies for Imaging, Radar and Communication Applications), which received funding from the European Union's Horizon 2020 research and innovation programme under Marie Skłodowska-Curie Innovative Training Network (ITN) grant agreement No. 765426. (Corresponding author: Davide Cimbri.)

Davide Cimbri, Jue Wang, Abdullah Al-Khalidi, and Edward Wasige are with the High-Frequency Electronics group, division of Electronics and Nanoscale Engineering, James Watt School of Engineering, University of Glasgow, G12 8LT, Glasgow, United Kingdom (e-mail: davide.cimbri@glasgow.ac.uk).

Color versions of one or more of the figures in this article are available online at

Digital Object Identifier terabits per second $(\mathrm{Tb} / \mathrm{s})$ wireless communication links are expected within the next ten years [9], in what will be the sixth generation (6G) networks [10]. In order to meet these performance levels, an increase in the bandwidth by several tens of gigahertz $(\mathrm{GHz})$ is required [11]. However, this can be achieved only through the exploitation of higher frequency spectrum regions, specifically, the terahertz (THz) band [12].

Indeed, recent technological innovations [13] regarding $\mathrm{THz}$ system components [14] indicate the viability of $\mathrm{THz}$ wireless communications [15]. Regarding transmitters (Tx), two main approaches for $\mathrm{THz}$ signal generation are currently employed: photonic and electronic techniques [16] [17]. The photonic approach has proven to be effective to achieve data rates of up to $100 \mathrm{~Gb} / \mathrm{s}$ along several meters long links [18]-[23], since telecom-based photonic components, such as laser diodes, modulators, and photo-diodes (PD), are available, together with low-loss optical fibre cables. Generally, this approach consists of photo-mixing two optically selected wavelengths by means of a high-speed PD, such as a uni-travelling-carrier photo-diode (UTC-PD) [24], to generate a $\mathrm{THz}$ carrier wave signal through optical heterodyne down-conversion [25]. However, this technology still remains too complex for portable consumer applications, such as mobile phones.

Regarding the electronic approach, there are several solidstate semiconductor-device candidates for $\mathrm{THz}$ emitters operating at room temperature (RT), including tunnel transittime (TUNNETT) diodes [26], impact ionization avalanche transit-time (IMPATT) diodes [27], Gunn diodes [28] [29], Schottky barrier diodes (SBD) [30], superlattice electronic devices (SLED) [31], transistors [32], and resonant tunnelling diodes (RTD) [33]. Complementary metal-oxidesemiconductor (CMOS) field-effect transistor (FET), silicon germanium (SiGe) heterojunction bipolar transistor (HBT) and bipolar CMOS (BiCMOS), indium phosphide (InP) HBT, and InP high-electron-mobility transistor (HEMTs) technologies have shown maximum oscillation frequencies $f_{\max }$ of up to $\sim 450 \mathrm{GHz}[34], \sim 720 \mathrm{GHz}[35], \sim 1.1 \mathrm{THz}$ [36], and $\sim$ 1.5 THz [37], respectively, while InP RTDs have demonstrated values above $2 \mathrm{THz}$ [38].

Fig. 1 shows some of the most relevant reported output radio frequency $(\mathrm{RF})$ power versus operating frequency performances for $\mathrm{THz}$ solid-state integrated sources in the 0.2-2 $\mathrm{THz}$ range, including UTC-PD-based photo-mixers, transistorbased terahertz monolithic integrated circuits (TMIC) and RTD-based oscillators. It can be seen that all the three technologies can provide output powers up to the milliwatt (mW) threshold in the $300 \mathrm{GHz}$-band ( 275-325 GHz [39]), while both UTC-PD and RTD-based emitters have also demonstrated to work well above $600 \mathrm{GHz}$, and above $1 \mathrm{THz}$ with associated RF power of up to few microwatt $(\mu \mathrm{W})$. Indeed, TMICs oscil- 


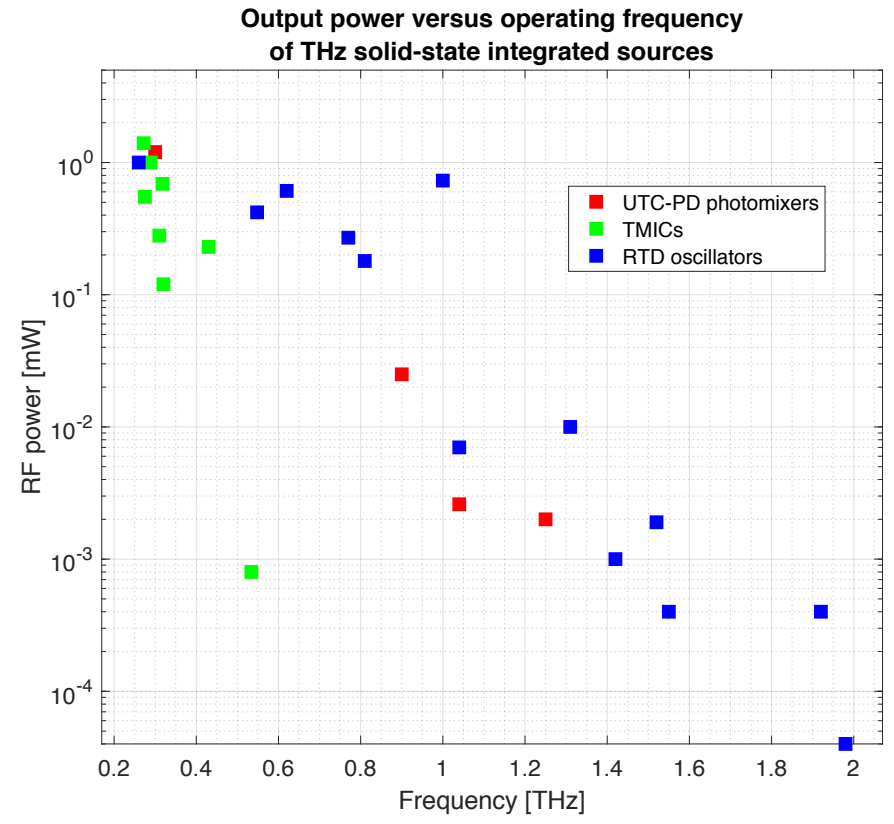

Fig. 1. Output power versus operation frequency of $\mathrm{THz}$ solid-state integrated sources, including UTC-PD-based photo-mixers, transistor-based TMICs and RTD-based oscillators in the $0.2-2 \mathrm{THz}$ range, outlining some of the most relevant results reported in the literature.

lators have shown to work at $\sim 300 \mathrm{GHz}$ in the fundamental mode [40], $\sim 430 \mathrm{GHz}$ in the second harmonic [41], and $\sim 540 \mathrm{GHz}$ in the third harmonic [42], and with operation frequencies of $1 \mathrm{THz}$ and $\mathrm{mW}$ output powers in the 0.3-0.9 $\mathrm{THz}$ range only enabled by extremely complex Tx designs, including frequency multiplier chains, push-push operation, sub-harmonic amplification stages, and array configurations [43]-[48], while RTD oscillators do not require any of these and are characterised by uncomplex circuit topologies, which drastically reduces production cost and increases integrability. Fig. 2 shows the performance of solid-state integrated $\mathrm{THz}$ wireless communication technologies with data rates of up to $100 \mathrm{~Gb} / \mathrm{s}$, including some of the most relevant literature results. As it is possible to notice, data rates of several tens of $\mathrm{Gb} / \mathrm{s}$ have been achieved by different technologies, including group IV (such as silicon $(\mathrm{Si})$ and $\mathrm{SiGe}$ ), and III-V semiconductors (such as gallium arsenide $(\mathrm{GaAs})$ and InP), even though the link distance is still very limited to well below $10 \mathrm{~m}$, with the longer ranges largely enabled by extremely high-gain antennas ( $>50 \mathrm{dBi})$, coherent receivers $(\mathrm{Rx})$, and power amplifier stages [49]. It is therefore clear that, in order to increase the link distance and realise practical $\mathrm{THz}$ wireless communications, a significant improvement in the performance of the associated compact semiconductorbased devices is required. From Fig. 2, it is also clear that RTD technology demonstrates comparable performance to other competing technologies in the context of ultra-broadband short-range wireless links. In addition, while the other technologies typically employ complex transmission signalling procedures, such as quadrature amplitude modulation (QAM), quadrature phase-shift keying (QPSK), etc., RTD-based systems have relied on simple amplitude modulation schemes

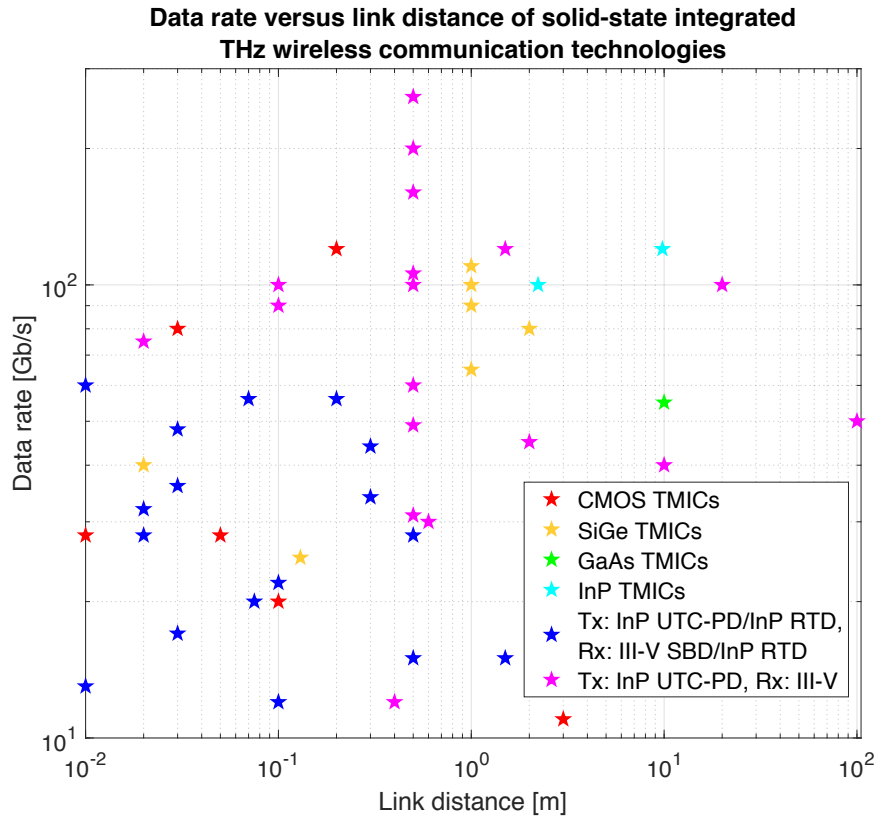

Fig. 2. Data rate versus link distance of solid-state integrated $\mathrm{THz}$ wireless communication technologies, outlining some of the most relevant literature results.

to achieve comparable speed performance, including on-off keying (OOK) and amplitude shift keying (ASK). Indeed, even though complex signalling, such as 16-QAM and higher constellations, feature high-spectral density, signal processing and synchronisation becomes mandatory, which makes the designed systems energy-hungry, especially at the Rx side. On the other hand, RTD transceivers (TRx) are characterised by simpler implementations, mainly square-law direct detectors, for which heavy signal processing is not required.

This review paper focuses on RTD technology for $\mathrm{THz}$ wireless communications, which features the simplest Tx and $\mathrm{Rx}$ circuit architectures among available $\mathrm{THz}$ technologies. The paper is organised as follows. Section II provides a description of the device principle of operation, technology, and modelling. Typical device designs approaches for THz emitters are also included, together with a succinct description of RTD oscillator design. Sections III describes the performance of reported THz RTD oscillators, while Section IV briefly reports RTD-based $\mathrm{THz}$ detectors. $\mathrm{THz}$ wireless links utilising the RTD at the Tx, Rx, as well as those employing all-RTD-based TRx, are described in Section V. The paper concludes with a discussion about the current technological challenges and futures perspectives in Section VI.

\section{RESONANT TUNNELLING DIODE}

\section{A. Principle of operation and technology}

The resonant tunnelling diode (RTD) is a one-dimensional (1D) vertical transport unipolar two-terminals semiconductor active device which is characterized by a highly non-linear current-voltage $(I V)$ characteristic, usually comprising of a negative differential resistance (NDR) region and two positive differential resistance (PDR) regions [50], as depicted in Fig. 


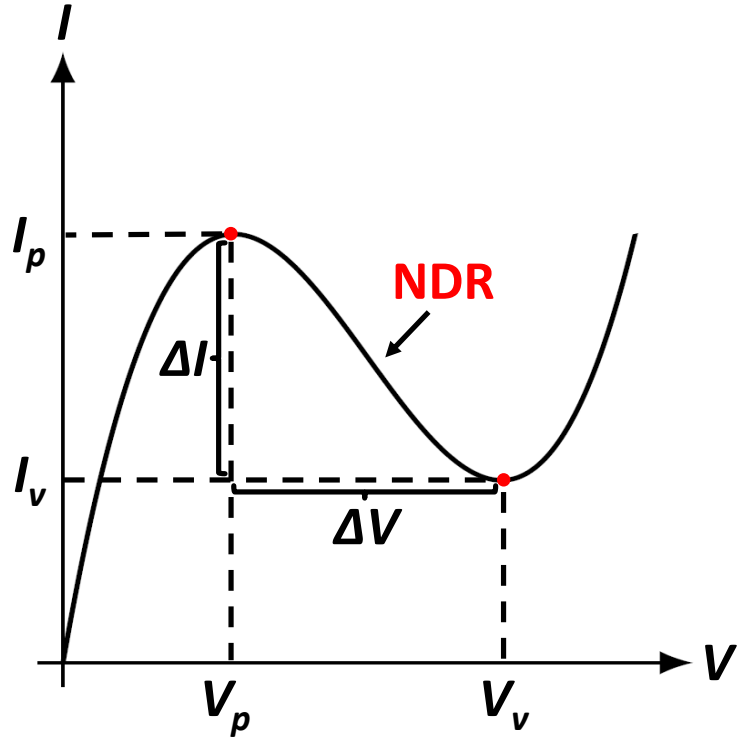

Fig. 3. Schematic illustration of the RT $I V$ characteristic of a generic RTD device, assuming a first and a second quasi-bound resonant level in the quantum well.

TABLE I

RTD III-V ELECTRONIC PHYSICAL PARAMETERS*

\begin{tabular}{ccccc}
\hline \hline Material & $m_{e}^{*}\left[m_{0}\right]$ & $E_{g}[\mathrm{eV}]$ & $\epsilon_{r, 0}\left[\epsilon_{0}\right]$ & $\Delta E_{c}[\mathrm{eV}]$ \\
\hline $\mathrm{GaAs}$ & 0.063 & 1.424 & 12.90 & $-0.28^{[a]}$ \\
\hline $\mathrm{AlAs}$ & 0.146 & $\begin{array}{c}2.949 \\
2.153^{* *}\end{array}$ & 10.06 & $0^{[a]}$ \\
& & 0.354 & 15.15 & $-1.35^{[b]}$ \\
\hline $\mathrm{InAs}$ & 0.023 & 2.386 & 12.04 & $0^{[b]}$ \\
\hline $\mathrm{AlSb}$ & 0.140 & 3.440 & 8.90 & $-2.00^{[c]}$ \\
\hline $\mathrm{GaN}$ & 0.200 & 6.130 & 9.14 & $0{ }^{[c]}$ \\
\hline $\mathrm{AlN}$ & 0.400 & $1.424+$ & $12.90-$ & $0.47-$ \\
\hline $\mathrm{Al}_{x} \mathrm{Ga}_{1-x} \mathrm{As}$ & $0.063+$ & $1.155 x+$ & $2.84 x$ & $0.33 x+$ \\
$(0.45 \leq x \leq 1)$ & $0.083 x$ & $0.370 x^{2}$ & & $0.14 x^{2[d]}$ \\
\hline $\mathrm{In}_{1-x} \mathrm{Ga}_{x} \mathrm{As}$ & $0.023+$ & $0.354+$ & $15.15-$ & $-4.88+$ \\
$(x \leq 0.47)$ & $0.035 x+$ & $0.593 x+$ & $2.87 x+$ & $0.81 x^{[a]}$ \\
& $0.009 x^{2}$ & $0.477 x^{2}$ & $0.67 x^{2}$ & \\
\hline $\mathrm{In}_{0.53 \mathrm{Ga}_{0.47} \mathrm{As}}$ & 0.042 & 0.738 & 13.90 & $-1.04^{[a]}$ \\
\hline \hline
\end{tabular}

${ }^{*}$ Some of the RT material parameters at the $\Gamma$ point [59]-[62]. $\mathrm{Al}_{x} \mathrm{Ga}_{1-x} \mathrm{As}$ : aluminium gallium arsenide, AlSb: aluminium antimonide, InAs: indium arsenide. $x$ is the molar fraction of the associated binary compounds. $m_{0} \simeq 9.11 \times 10^{-31} \mathrm{~kg}$ is the electron rest mass, while $\epsilon_{0} \simeq 8.854 \times 10^{-12}$ $\mathrm{F} / \mathrm{m}$ is the vacuum permittivity. $\Delta E_{c}$ is given with respect to a reference material $(0 \mathrm{eV}):[a]:$ AlAs, $[b]:$ AlSb, $[c]:$ AlN, $[d]:$ GaAs. ${ }^{* *} X$ point value. These parameters are generic and strain-independent.

3. This non-linearity arises as a result of the quantum mechanical resonant tunnelling [51] of electrons/holes through the device, even though electron operation is typically preferred due to the higher associated drift mobility, which leads to higher current density and maximum operation frequency. The precise shape of the $I V$ characteristic depends on different factors, such as device size, material composition, epitaxial structure, and temperature [52] [53]. The NDR region is

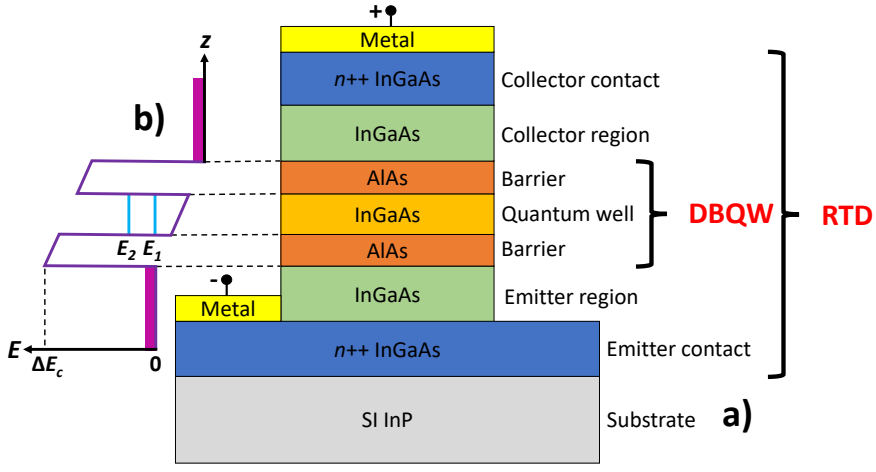

Fig. 4. In a), a sketch of the typical layer structure of a generic double-barrier RTD device, outlining the DBQW region. In b), the corresponding simplified conduction band diagram in forward bias (peak resonance condition). The quasi-bound resonant levels $E_{1}$ and $E_{2}$ are quantised due to the $\mathrm{QW}$ discrete energy spectrum.

characterised by $I_{p}$ and $I_{v}$, which are the peak and valley currents, respectively, and corresponding voltages $V_{p}$ and $V_{v}$. Further, it can be described by $\Delta I=I_{p}-I_{v}$, which is the peak-to-valley current difference, $\Delta V=V_{p}-V_{v}$, the peakto-valley voltage difference, and PVCR $=I_{p} / I_{v}$, the peakto-valley current ratio. The electrical span of the NDR region determines the theoretical maximum RF power the RTD device can deliver to a load [54]. The actual RF output power would depend on different factors, such as the operation frequency, device and circuit parasitic elements, and impedance matching considerations [55].

RTD devices realized in III-V compound semiconductors show attractive characteristics for $\mathrm{THz}$ operation [56] [57]. The electronic physical parameters of commonly used III$\mathrm{V}$ materials employed to design RTD-based $\mathrm{THz}$ sources are shown in Table I, including the effective electron mass $m_{e}^{*}$, energy band-gap $E_{g}$, conduction band offset $\Delta E_{c}$, and static relative dielectric constant $\epsilon_{r, 0}$ at RT $(300 \mathrm{~K})$. In general, small $m_{e}^{*}$ leads to high drift mobility and improved transport properties, whereas high $\Delta E_{c}$ improves the PVCR by suppressing thermionic emission across the device structure. Even though first attempts in $\mathrm{THz}$ oscillators realisation were based on GaAs [58], the most dominant RTD technology for $\mathrm{THz}$ applications nowadays is based on the indium gallium arsenide/aluminium arsenide (InGaAs/AlAs) material system [63], which is epitaxially grown onto a lattice-matched semi-insulating (SI) InP substrate through either molecular beam epitaxy (MBE) or metal-organic vapour phase epitaxy (MOVPE). This is because of the low $m_{e}^{*}$ of $\operatorname{In}_{0.53} \mathrm{Ga}_{0.47} \mathrm{As}$ (RT saturation drift velocity $v_{e, s} \sim 8 \times 10^{6} \mathrm{~cm} / \mathrm{s}$ [64]) and high $\Delta E_{c}$, together with a low specific contact resistivity $\rho_{c} \sim$ $10^{-8} \Omega \mathrm{cm}^{2}$ [65], which enhance current density and $f_{\max }$. Recently, III-nitrides have also gained interest in the RTD community [66]-[68]. They feature large $\left|\Delta E_{c}\right|(\sim 2.0 \mathrm{eV}$ in the gallium nitride/aluminium nitride (GaN/AlN) material system), good $v_{e, s}\left(\sim 2.0 \times 10^{7} \mathrm{~cm} / \mathrm{s}\right.$ for $\left.\mathrm{GaN}\right)$, and highbreakdown voltage. Despite that, they are characterised by large $m_{e}^{*}\left(\sim 0.2 m_{0}\right.$ for $\mathrm{GaN}$ and $\sim 0.4 m_{0}$ for AlN $)$, the Ohmic contacts are poor $\left(\rho_{c} \sim 10^{-5} \Omega \mathrm{cm}^{2}\right)$, and the epitaxial growth is still immature. Demonstrated devices have exhibited 


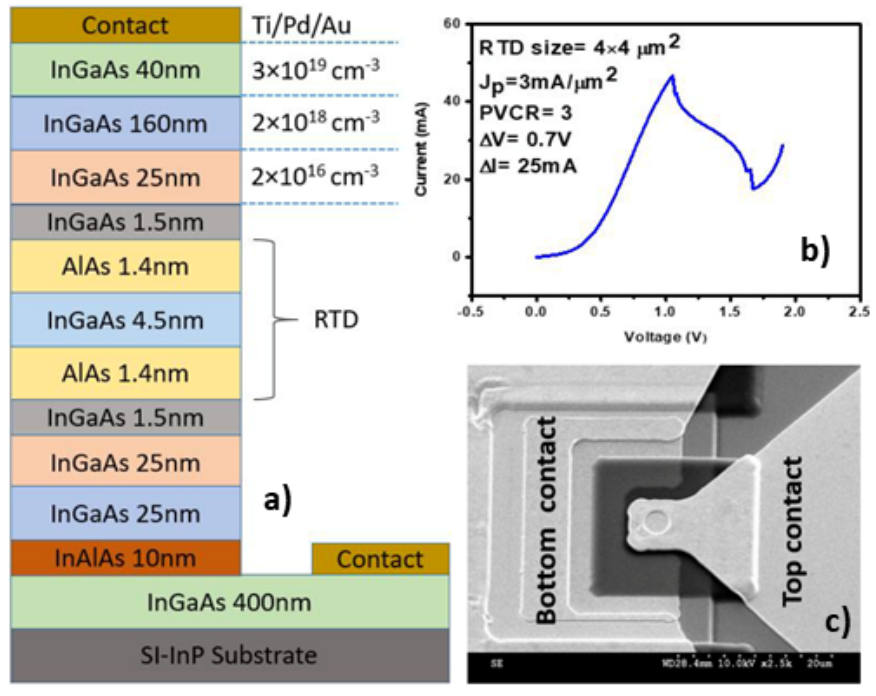

Fig. 5. In a), a schematic illustration of the RTD epitaxial structure employed in the realisation of the $260 \mathrm{GHz}$ oscillator, in b), the measured static $I V$ characteristic of a fabricated $16 \mu \mathrm{m}^{2}$ large device, in c), a SEM image of the fabricated device (adapted and reprinted from [83] with permission).

$f_{\max }$ of under $200 \mathrm{GHz}$, so the jury is still out for this material system for $\mathrm{THz}$ sources.

Fig. 4 a) shows a typical layer structure of a $n$-type intraband double-barrier RTD device in the InGaAs/AlAs material system. The core of the diode comprises of a low band-gap (InGaAs) quantum well (QW) layer sandwiched by two high band-gap (AlAs) barrier layers, forming the so-called double barrier quantum well (DBQW). Often, either an indium (In)rich InGaAs well [69] or an indium arsenide (InAs) sub-well [56] is used for very high-speed operation. The device features InGaAs-based undoped/lightly-doped spacer layers on either emitter and collector sides, together with $n+$ emitter/collector regions and heavily-doped contacts. Spacers are designed to avoid dopant diffusion into the DBQW active region, to reduce the device self-capacitance, and to maximise both speed and power performances [70]-[72], while contacts doping level and In molar fraction are optimised to enhance current density and reduce the associated contact resistance [69]. Generally speaking, layers thickness/doping level and QW indium molar fraction reflect on the diode $I V$ characteristic and capacitance, and therefore impedance [52] [73], affecting both speed and RF power performances. Variations in the DBQW, spacers and contacts are found among the different RTDs reported in the literature, while metal contacts typically employ a titanium/palladium/gold ( $\mathrm{Ti} / \mathrm{Pd} / \mathrm{Au})$ stack scheme.

The DBQW region is nanometric in dimensions, typically well below $10 \mathrm{~nm}$, and therefore thin enough to allow electron quantum mechanical resonant tunnelling [74]. In this sense, the term resonant refers to the behaviour of electrons with energy lower than the barrier potential, but still able to travel across the DBQW region [75]. This is a consequence of the wave-particle duality, where the electron can be described through a wave-function [76]. The energy states in the QW are quantised, i.e., the energy distribution spectrum is discrete due to the associated stair-like density of states (DOS) [77].

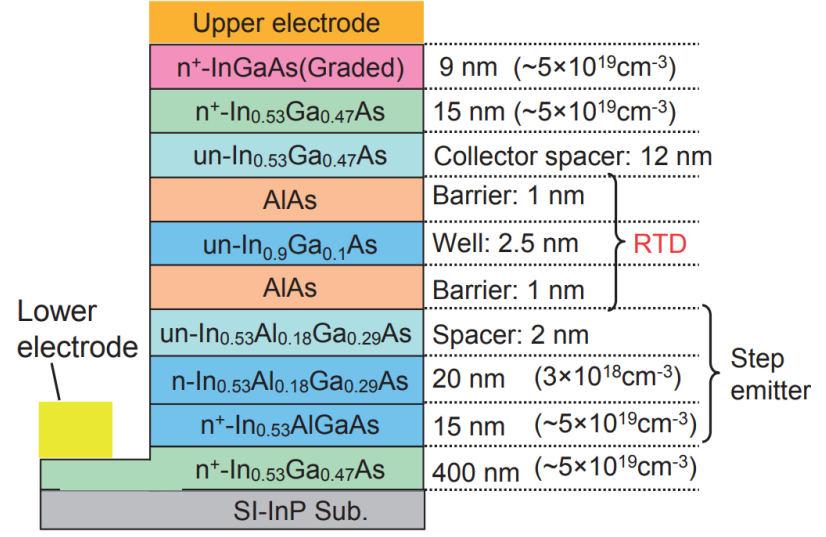

Fig. 6. Schematic illustration of the RTD epitaxial structure design employed in the realisation of the $1.92 \mathrm{THz}$ [84] and $1.98 \mathrm{THz}$ [38] oscillators (reprinted from [84] with permission).

The simplified conduction band diagram of a generic RTD device under forward bias (peak resonant condition) is depicted in Fig. 4 b). In the illustration, the first and second quasi-bound resonant energy levels $E_{1}$ and $E_{2}$ are shown. The probability of electron tunnelling through the DBQW is defined by the transmission coefficient $T_{d b q w}$ : at the resonant condition, $T_{d b q w} \approx T_{1} T_{2} \sim 1$, whereas $T_{d b q w} \sim 0$ otherwise, where $T_{1}$ and $T_{2}$ are the transmission coefficients associated with the first and second barrier, respectively. The resonant condition is met when the electrons entering the DBQW region from the emitter conduction band have energy equal to one of the allowed QW energy levels [78]. Thus, as the electron transmission coefficient changes with the applied bias voltage, the device static $I V$ characteristic exhibits a NDR [79]. Moreover, since resonant tunnelling in these 1D vertical transport semiconductor-based nanostructures is a very fast process, the NDR is characterized by an extremely wide bandwidth, which can extend up to the $\mathrm{THz}$ range [80]-[82]. Therefore, RTDs can be embedded in resonators to build ultrahigh speed $\mathrm{THz}$ continuous wave $(\mathrm{CW})$ sources and highly sensitive detectors.

An example of an RTD epitaxial layers structure used in THz sources up to around $300 \mathrm{GHz}$ [83] is depicted in Fig 5 a). It comprises of a $\mathrm{In}_{0.53} \mathrm{Ga}_{0.47} \mathrm{As} / \mathrm{AlAs} \mathrm{DBQW}$ heterostructure with $\sim 1.46 \mathrm{~nm}$ thick barriers, $\sim 4.4 \mathrm{~nm}$ thick $\mathrm{In}_{0.53} \mathrm{Ga}_{0.47} \mathrm{As}$ QW, and $25 \mathrm{~nm}$ thick lightly-doped (Si: $2 \times 10^{16} \mathrm{~cm}^{-3}$ ) spacers, which was grown onto a SI InP substrate by MBE. The reported RTD electrical quantities of a fabricated device with mesa area $A \sim 16 \mu \mathrm{m}^{2}$ are reported in Fig. 5 b), together with the measured static $I V$ characteristic, and were peak current density $J_{p}=I_{p} / A \simeq 3 \mathrm{~mA} / \mu \mathrm{m}^{2}, \Delta I \simeq 25 \mathrm{~mA}$, $\Delta V \simeq 0.7 \mathrm{~V}$, and $\mathrm{PVCR} \simeq 3$. A scanning electron microscope (SEM) image of the fabricated device is shown in Fig. 5 c). Fig. 6 shows a high-speed InGaAs/AlAs DBQW heterostructure which has been used in $>1 \mathrm{THz}$ sources [38] [84]. It features an indium aluminium gallium arsenide (InAlGaAs)based graded emitter layer, which allows to reduce the DC voltage needed to bias the RTD device in its NDR region by moving the emitter conduction band edge closer to $E_{1}$, thereby shifting the peak voltage $V_{p}$ to lower bias [85]. This increases 


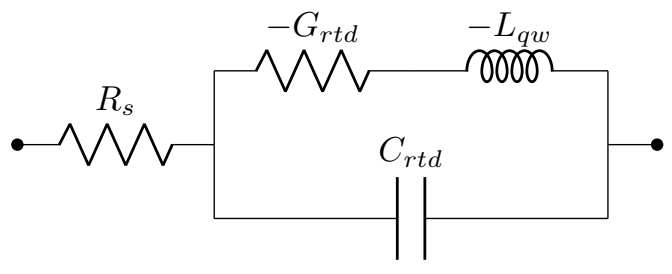

Fig. 7. Small-signal equivalent circuit of an RTD device [95].

the DC-to-RF efficiency, reduces thermal heating, and prevents the generation of high-electric fields in the diode depletion regions, which could cause electrical breakdown. An In-rich $\mathrm{In}_{0.9} \mathrm{Ga}_{0.1} \mathrm{As} \mathrm{QW}$ was employed to further reduce the DC bias by depressing the ground state $\left(E_{1}\right)$ subband, while the second subband $\left(E_{2}\right)$ stays almost in place, increasing the PVCR [69]. Moreover, employing thin $\sim 1 \mathrm{~nm}$ thick AlAs barriers and a thin $\sim 2.5 \mathrm{~nm}$ thick QW increases current density and reduces the electron transit time [86], increasing $f_{\max }$, while a 12 $\mathrm{nm}$ thick undoped collector spacer layer was used to trade-off between depletion and carrier transit-related capacitances [71] [87]. The heavily doped In-rich graded cap layer was designed to improve the Ohmic top contact by reducing the Schottky barrier height and the associated contact resistance. The $I V$ curve electrical quantities of $\mathrm{a} \sim 0.2 \mu \mathrm{m}^{2}$ large fabricated device were reported in [38] and are $J_{p} \simeq 31 \mathrm{~mA} / \mu \mathrm{m}^{2}$, $\Delta I \simeq 2.8 \mathrm{~mA}, \Delta V \simeq 0.5 \mathrm{~V}$, and $\mathrm{PVCR} \simeq 1.8$.

\section{B. Modelling}

The non-linear $I V$ characteristic of an RTD is usually computed through quantum mechanical analytical expressions derived from semiconductor device physics [88] [89] or through advanced numerical techniques, such as the nonequilibrium Green's function method [90]. Other modelling approaches include fitting experimental data [91] [92] or, for simplified device analysis, employing a simple cubic function, where $I(V) \propto-V\left(1+V^{2}\right)$ [54], or higher order polynomials [94]. However, at any specific bias point, the RTD can be modelled using a linear small-signal lumped element equivalent circuit model [95], as shown in Fig. 7. In this representation, the device is modelled through a selfcapacitance $C_{r t d}=C_{d}+C_{q w}+C_{t}$, where $C_{d}=\epsilon_{r t d} A / t_{r t d}$ is the depletion capacitance (being $\epsilon_{r t d}$ and $t_{r t d}$ the equivalent dielectric constant and thickness associated with the RTD depleted regions, respectively, while $A$ is the RTD mesa area), while $C_{q w} \approx \tau_{c} G_{r t d}=\hbar G_{r t d} / \Gamma_{c}$ and $C_{t} \approx \tau_{t} G_{r t d}$ are the quantum capacitance [96] and the capacitance associated with depletion regions transit delay [97] (being $\hbar$ the normalised Plank constant, $\Gamma_{c}$ the QW resonant level full width at half maximum (FWHM) energy broadening as a result of electron wave-function leakage from the collector barrier, and $\tau_{t}$ the depletion regions electron transit time), respectively, in parallel with the series of a negative differential conductance $-G_{r t d}$ and a negative $\mathrm{QW}$ inductance $L_{q w} \approx-\tau_{q w} / G_{r t d}$ [98], with $G_{r t d}$ positive in the NDR region. In particular, $L_{q w}$ models the lag associated with the QW charging and discharging caused by the change in the available emitter electron density for resonant tunnelling with bias, being related to the $\mathrm{QW}$ electron quasi-bound state tunnelling lifetime $\tau_{q w}=\tau_{e} \tau_{c} /\left(\tau_{e}+\tau_{c}\right)$ [95] [98] (where $\tau_{e}$ and $\tau_{c}$ are the inverse of the electron QW-toemitter and QW-to-collector escape rates, respectively), $C_{q w}$ is associated with the consequent change in the collector charge due to the QW charge variation with the applied voltage [96], while $G_{r t d}$ models the RF gain capability of the device [99]. In principle $G_{r t d}, C_{r t d}$, and $L_{q w}$ are both voltage and frequency dependent. High and low-frequency analytical expressions for the RTD admittance have been provided in [55] [100]. The model is completed by a series resistance $R_{s}$, which mainly models the emitter/collector contact resistance $R_{c}$, the sheet resistance of the device mesa, spreading resistance, and the resistance associated with emitter/collector contacts and bondpads metallisation [101]. An equivalent $R C$ model based on the total intrinsic delay time across the RTD structure $\tau_{r t d}=\tau_{d b q w}+\tau_{t} / 2$, has been proposed in [55] [102] (being $\tau_{d b q w}$ the tunnelling time across the DBQW region), together with advanced $R L C R$ models [52] [100] [103] [104].

Both the device measured static $I V$ characteristic and its small-signal model elements can be utilised for technological optimisation. For instance, they can be used to estimate the device maximum RF power capability and cut-off frequency. For practical device equivalent circuit modelling, $f_{\max }$ can be approximated, as [98] [101]:

$$
f_{\max } \approx \frac{G_{r t d}}{2 \pi C_{r t d}} \sqrt{\frac{1}{R_{s} G_{r t d}}-1}
$$

which corresponds to the frequency at which the NDR is cancelled out by the equivalent circuit resistance. Here, $C_{r t d}$ and $G_{r t d}$ can be either extracted from S-parameters measurements [105] or theoretically estimated, where $G_{r t d} \approx$ $3 \Delta I / 2 \Delta V=3 A \Delta J / 2 \Delta V$ [94], with $\Delta J=\Delta I / A$ the available current density. The resistance $R_{s}$ can be both extracted from measured S-parameters or estimated as $R_{s} \approx R_{c}=$ $\rho_{c} / A$, where the specific contact resistivity $\rho_{c}$ is extracted from transfer length model (TLM) measurements [106]. However, S-parameters characterisation of the RTD device remains nontrivial [107], especially at $\mathrm{THz}$ frequencies $(>100 \mathrm{GHz})$ due to parasitic bias oscillations [108] [109]. Accurate characterisation with reliable de-embedding for small-signal parameters extractions has been demonstrated up to $110 \mathrm{GHz}$ [105].

A reliable non-linear model for the RTD device is yet to be developed to efficiently carry out oscillator large-signal [98] analysis, e.g., to estimate the output power at the oscillation frequency, or in the design of RTD-based coherent detectors [52], but work in this direction is underway [110]-[112]. $C_{d c}$. In summary, current RTD oscillator design is carried out relying on empirical approaches [55] [94]. Based on the measured static $I V$ characteristics and extracted small-signal parameters of the devices, basic oscillator design is performed and, after experimental characterisation, circuit optimisation is carried out.

\section{RTD THz oscillator design}

RTD-based NDR oscillators can produce either sinusoidal or non-sinusoidal waveforms. The latter, called relaxation oscillators, have been demonstrated in both transmission line 


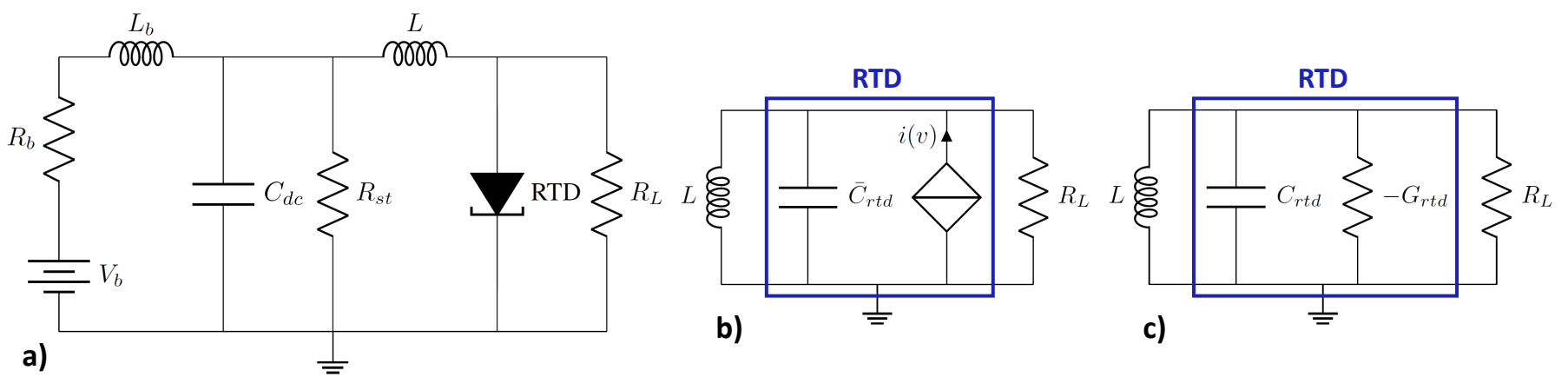

Fig. 8. In a), the RTD-based sinusoidal oscillator lumped-element equivalent circuit topology. In b), the oscillator RF large-signal equivalent circuit. In c), the oscillator simplified equivalent circuit at start-up.

[113] or monolithic [114] forms, but they typically work at lower frequencies and so they are not discussed here. On the other hand, state-of-the-art RTD THz Tx exclusively employ sinusoidal generators, whose principle of operation conforms to classical NDR diode-based electronic oscillators. When the RTD is biased within the NDR region, electronic noise in the circuit is amplified by the NDR and the system filters out all frequency components, except those defined by the resonator passband. The shape of the spectrum defines its quality factor $(Q)$ and the corresponding relative bandwidth, which depend upon the resistor-inductor-capacitor $(R L C)$ lossy resonator. If the large-signal NDR can compensate for circuit, device and load (antenna) losses within the resonator bandwidth, a stable oscillating signal is obtained across the load [115]. An RTD oscillator can be therefore considered a DC-to-RF power converter, where the energy provided by the DC bias supply is transformed into an RF output signal with a certain efficiency, and then delivered to a load, usually an antenna.

A schematic representation of a generic RTD-based sinusoidal oscillator lumped-element equivalent circuit topology is shown in Fig. 8 a), together with its large signal RF equivalent circuit (Fig. 8 b)) and at start-up (Fig. 8 c)). The DC part of the circuit is composed of the DC bias supply $V_{b}$, the bias line, which is modelled through its parasitic resistance $R_{b}$ and inductance $L_{b}$, the decoupling capacitance $C_{d c}$, and the stabilizing shunt resistance $R_{s t}$, while the RF part of the circuit comprises of the resonating inductance $L$, the RTD device, and the load resistance $R_{L}=1 / G_{L}$. The shunt resistor suppresses lowfrequency bias oscillations and since the DC bias is fed via the resonating inductance, the decoupling capacitor is used to ground the inductor and to short-circuit the stabilising resistor at the oscillation frequency $f_{\text {osc }}$, thereby decoupling the oscillator circuit from the DC bias supply. The decoupling capacitor is designed to be a short-circuit at $f_{\text {osc }}$ [94]:

$$
f_{\text {osc }} \approx \frac{\sqrt{\left(L-C_{r t d} R_{s}^{2}\right)}}{2 \pi L \sqrt{C_{r t d}}\left(1+R_{s} G_{L}\right)}
$$

(which simplifies to $\approx 1 / 2 \pi \sqrt{L C_{r t d}}$ if $R_{s} \rightarrow 0$ [98]), i.e., $1 / 2 \pi f_{o s c} C_{d c} \rightarrow 0$, while the shunt resistor is designed so that $R_{s t}>1 / G_{r t d}$ [116]. Note that $R_{s t}$ establishes the maximum device size $A_{\max }=2 \Delta V / 3 \Delta J R_{s t}$ which can be used to realise an oscillator [117]. The resonating inductance $L$ is usually realised from a short section of a transmission line, such as

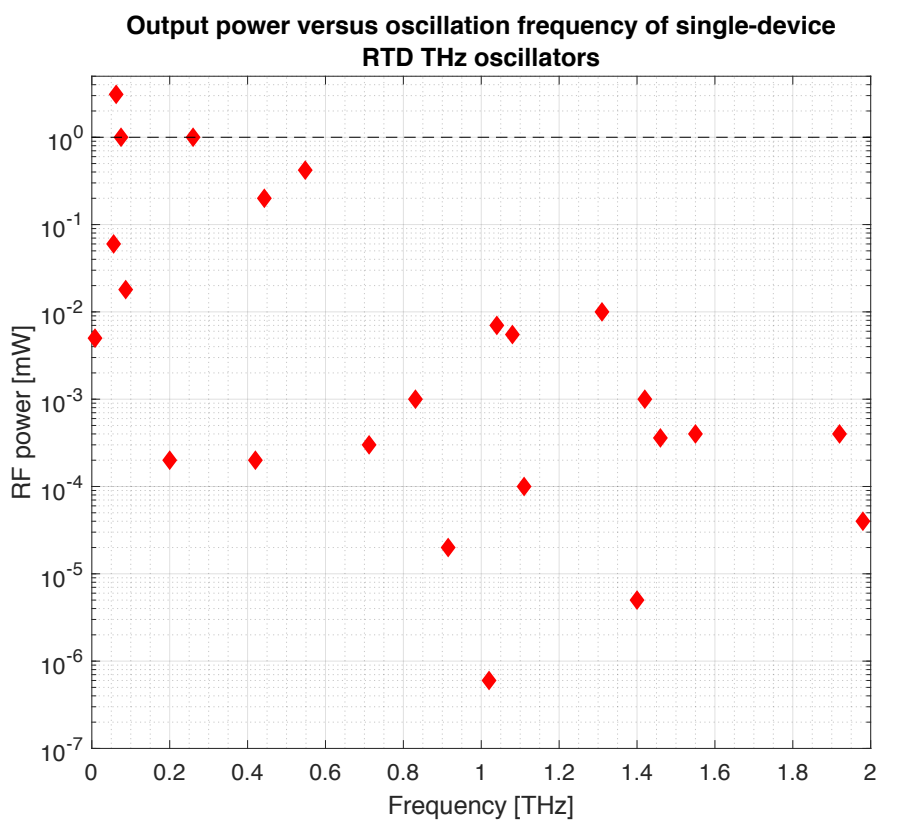

Fig. 9. Output power versus fundamental frequency of oscillation for some of the most relevant reported single-device RTD $\mathrm{THz}$ oscillators to date, including some results below $100 \mathrm{GHz}$.

a coplanar waveguide (CPW) [118], a coplanar stripline (CPS) [119], or a microstrip line [120], where $2 \pi f_{o s c} L=Z_{0} \tan (\beta l)$, being $\beta$ the phase constant, $l$ the length of the stub, and $Z_{0}$ its characteristic impedance, while the resonating capacitance is provided by the intrinsic self-capacitance of the diode. This is often the case for RTD oscillators operating below $\sim 300$ $\mathrm{GHz}$, where an external load is employed [83]. For higher oscillation frequencies, an on-chip integrated antenna (mostly slot) is usually employed, which works both as the resonating inductance as well as radiator, i.e., load resistance [33].

The maximum maximum oscillator output power $P_{R F, \max }$ can be expresses as [54]:

$$
P_{R F, \max } \approx \frac{3}{16} A \Delta J \Delta V=\frac{3}{16} \Delta I \Delta V
$$

However, in practical oscillators, the delivered output power at the frequency of oscillation $P_{R F, \text { out }}$ is much lower (with factors that can range from 3-4 up to several hundreds depending on the operation frequency), mostly due to the RTD device 
TABLE II

RTD DEVICES ELECTRICAL QUANTITIES

\begin{tabular}{|c|c|c|c|c|c|c|c|}
\hline Ref. & $J_{p}\left[\mathrm{~mA} / \mu \mathrm{m}^{2}\right]$ & $\Delta J\left[\mathrm{~mA} / \mu \mathrm{m}^{2}\right]$ & PVCR [ ] & $V_{p}[\mathrm{~V}]$ & $C_{r t d}\left[\mathrm{fF} / \mu \mathrm{m}^{2}\right]$ & $\rho_{c}\left[\Omega \mu \mathrm{m}^{2}\right]$ & $f_{\max }{ }^{*}$ \\
\hline$[84]$ & 50 & 20.6 & 1.7 & 0.4 & - & - & - \\
\hline$[38]$ & 31 & 13.8 & 1.8 & - & - & - & - \\
\hline [121] & - & 14 & - & - & - & 3 & - \\
\hline [127] & 29 & 14.5 & 2 & - & - & - & - \\
\hline [92] & 1.9 & 1.1 & 2.5 & 0.9 & 2.5 & 83 & $317 \mathrm{GHz}$ \\
\hline [93] & 1.2 & 1.1 & 12 & 1.4 & 4.5 & 106 & $114 \mathrm{GHz}$ \\
\hline [126] & 6.9 & 2 & 1.4 & 0.7 & 8.1 & 4.6 & $1.08 \mathrm{THz}$ \\
\hline [128] & 6.7 & 1.9 & 1.4 & 0.6 & - & - & - \\
\hline
\end{tabular}

*Computed through Eq. (2). " - " stands for not provided.

TABLE III

RTD THZ OSCILLATORS SPECIFICS

\begin{tabular}{|c|c|c|c|c|c|c|c|}
\hline Ref. & $A\left[\mu \mathrm{m}^{2}\right]$ & $\Delta I[\mathrm{~mA}]$ & $\Delta V[\mathrm{~V}]$ & $P_{R F, \max }[\mathrm{mW}]$ & Antenna & $f_{\text {osc }}$ & $P_{R F, \text { out }}{ }^{*}$ \\
\hline$[84]$ & 0.1 & 2.1 & 0.4 & 0.2 & slot & $1.92 \mathrm{THz}$ & $0.4 \mu \mathrm{W}$ \\
\hline [121] & 1.4 & 19.6 & 0.4 & 1.5 & slot & $620 \mathrm{GHz}$ & $0.6 \mathrm{~mW}^{[a]}$ \\
\hline [127] & 0.5 & 7.2 & 0.3 & 0.4 & dipole & $1 \mathrm{THz}$ & $0.7 \mathrm{~mW}^{[b]}$ \\
\hline$[92]$ & 16 & 18.2 & 0.8 & 2.7 & I & $84 \mathrm{GHz}$ & $2 \mathrm{~mW}^{[c]}$ \\
\hline [93] & 26.4 & 29 & 1.3 & 7.1 & 1 & $62.5 \mathrm{GHz}$ & $3.1 \mathrm{~mW}$ \\
\hline [126] & 0.9 & 1.8 & 0.2 & 0.1 & patch & $1.52 \mathrm{THz}$ & $1.9 \mu \mathrm{W}^{[d]}$ \\
\hline [128] & 1.6 & 3 & 0.2 & 0.1 & dipole & $675 \mathrm{GHz}$ & $47 \mu \mathrm{W}^{[e]}$ \\
\hline
\end{tabular}

${ }^{*}$ Reported values are radiated when the antenna type is specified, on-chip measured otherwise. [a]: two-elements synchronised oscillators array, [b]: 89-element unsynchronised oscillators array, $[c]$ : two-parallel RTDs oscillator, $[d]$, triple-push configuration, $[e]$, differential double-RTD oscillator. " - " and " / " stand for not provided and not included, respectively.

negative differential conductance (NDC) roll-off caused by the intrinsic electron delay time across the RTD structure [52] [73] [102], but also due to extrinsic device and circuit parasitics [73] [117]. Indeed, a non-negligible $R_{s}$ causes the oscillator RF power to drop, since it poses a boundary to $f_{\max }[83]$. Approximate but reliable analytical expressions have been proposed to estimate the output power at a specific oscillation frequency due to NDC roll-off and $R_{s}$ constrains [55] [98] [117], but simple forms include $P_{R F, \text { out }} \approx P_{R F, \max } \cos \left(2 \pi f_{o s c} \tau_{r t d}\right)$ [121], and $P_{R F, \text { out }} \approx P_{R F, \max }\left[1-\left(f_{\text {osc }}^{2} / f_{\max }^{2}\right)\right]$ [122].

\section{RTD THZ OSCILLATORS}

Different approaches have been adopted to develop InPbased DBQW RTD THz oscillators, featuring different device epitaxial structures and circuit implementations. The details of state-of-the-art oscillators are summarised in Table II, Table III, and Fig. 9. The highest reported fundamental oscillation frequency is $\simeq 1.98 \mathrm{THz}$ with around $40 \mathrm{nW}$ of radiated output power [38], while the highest reported RF power (on-chip measured) is $\simeq 1 \mathrm{~mW}$ at $0.26 \mathrm{THz}$ [83]. Table II provides with the electrical quantities of the employed RTD devices, while Table III reports the oscillator specifics, where provided. Note that, for frequencies far beyond $300 \mathrm{GHz}$, oscillator design employs integrated antennas and the devices are characterised by high $J_{p}$ and low $\Delta V(<0.5 \mathrm{~V})$. Moreover, device sizes are small (around or well below $1 \mu \mathrm{m}^{2}$ ) and the RF power is typically low ( $\mu \mathrm{W}$ range). On the other hand, in the lowTHz band, moderate $J_{p}$, large $\Delta V(\gg 0.5 \mathrm{~V})$, and large mesa area $\left(\geq 16 \mu \mathrm{m}^{2}\right)$ devices are employed, as well as external antenna loads, with output powers in the $\mathrm{mW}$ range. Indeed, $\Delta I$, as well as $\Delta V$, are high for devices operating below $600 \mathrm{GHz}$, while small for RTDs operating above $1 \mathrm{THz}$. From Table III, it can be seen that typical $C_{r t d}$ are in the range of 3-8 $\mathrm{fF} / \mu \mathrm{m}^{2}$, while $\rho_{c}$ are also very varied, ranging from 3-106 $\Omega \mu \mathrm{m}^{2}$, which mainly depends on the fabrication process of Ohmic contacts. The peak current density $J_{p}$ also 


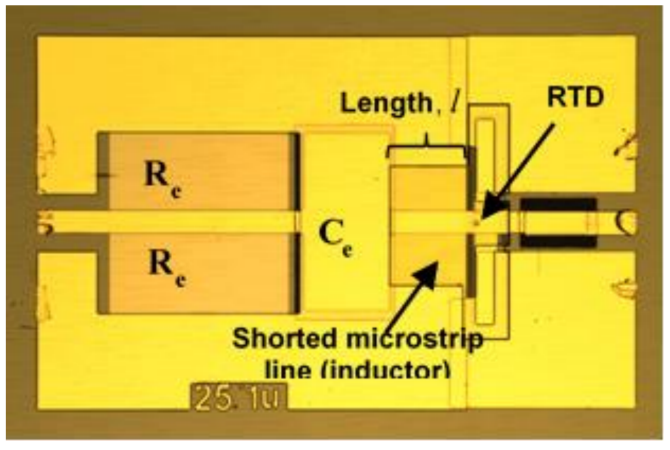

Fig. 10. Photomicrograph of the fabricated $260 \mathrm{GHz}$ microstrip RTD oscillator (reprinted from [83] with permission).

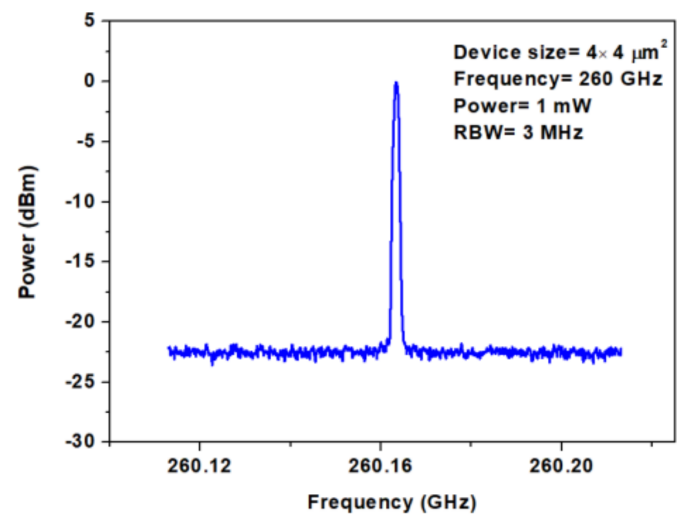

Fig. 11. Measured spectrum of the $260 \mathrm{GHz}$ microstrip RTD oscillator (reprinted from [83] with permission).

varies over a large range, between $1-50 \mathrm{~mA} / \mu \mathrm{m}^{2}$ which, in some ways, shows the relative immaturity of the epitaxial design approaches and associated oscillator circuit realisation. Fig. 9 shows the reported output power and corresponding $f_{\text {osc }}$ of these oscillators to date. Clearly, THz operation has been demonstrated, but the output power is still under $1 \mathrm{~mW}$ beyond $300 \mathrm{GHz}$, which is perhaps the main limitation of this technology. These results also show a wide variation in performance, which perhaps indicates the lack of established and optimal design practices.

A micrograph of the fabricated $0.26 \mathrm{THz}$ oscillator is shown in Fig. 10 and its spectrum of oscillations is shown in Fig. 11 [83]. The corresponding measured output power was $\simeq 1$ $\mathrm{mW}$, with a corresponding DC-to-RF efficiency of $\simeq 0.7 \%$. Circuit design was carried out employing a single RTD device and a $88 \mu \mathrm{m}$ long microstrip-based resonating inductance with characteristic impedance $Z_{0}=10.4 \Omega$, where Polyimide PI2545 was used as dielectric. It featured a $0.1 \mathrm{pF}$ silicon nitride $\left(\mathrm{SiN}_{x}\right)$ metal-insulator-metal (MIM) decoupling capacitor, a $1.3 \mathrm{pF} \mathrm{SiN}_{x}$-based MIM DC block capacitor, together with a nichrome (NiCr)-based stabilising resistor with $R_{s t}=22 \Omega$. The oscillator was designed for on-wafer probing and did not feature an on-chip antenna and microfabrication was carried out solely with standard low-cost optical-lithography.

RTD THz oscillators operating far beyond $300 \mathrm{GHz}$ employ integrated on-chip antenna loads with different antenna types, such as slot [84], Vivaldi [56], radial-line slot [123], patch

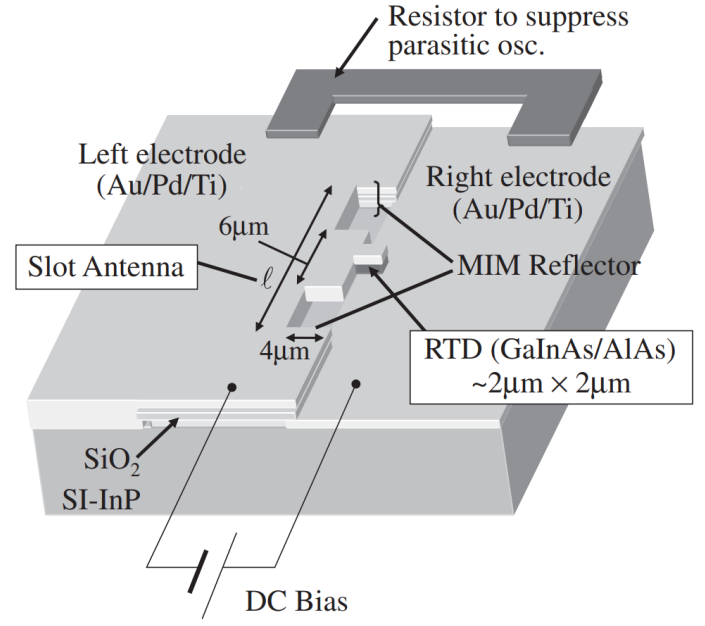

Fig. 12. Schematic representation of an RTD THz oscillator integrated with a slot-antenna (reprinted from [55] with permission).

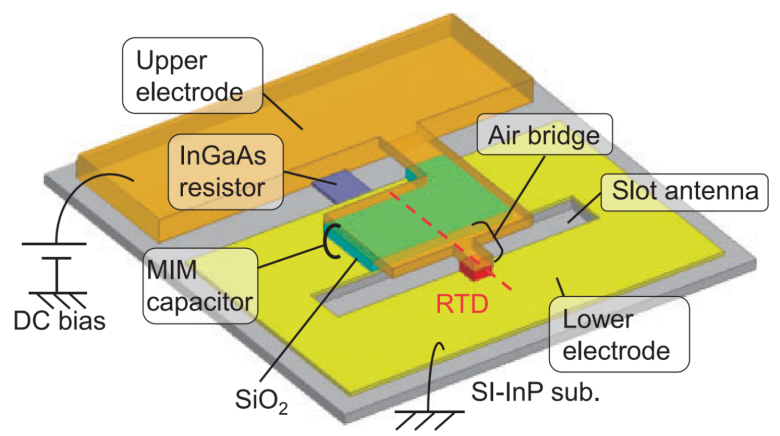

Fig. 13. Sketch of the circuit layout employed in the realisation of the 1.92 RTD THz oscillator (reprinted from [84] with permission).

[124]-[126], dipole [127]-[129], and bow-tie [130]. However, the most common on-chip antenna is the slot-antenna, which is used in conjunction with a hemispherical Si lens [131] [132]. Because of the high dielectric permittivity of InP $\left(\epsilon_{r, 0} \sim 12.5\right.$ [133], $\epsilon_{r, \infty} \sim 9.6$ [134] $)$, most of the output power is radiated into the substrate. Thus, the oscillator is mounted onto the Si lens to extract and collimate it, with the thickness of the chip and the lens designed to maximise the power extraction efficiency [69]. Fig. 12 shows the general schematic of a RTD THz oscillator integrated with a slotantenna [55]. In this configuration, the electrodes of the RTD device are connected to the left and right electrodes of the antenna, which have a silicon dioxide $\left(\mathrm{SiO}_{2}\right)$ layer between them, forming a decoupling MIM capacitor, across which a stabilising resistor is connected. The frequency of oscillation is mainly determined by the parallel resonance of $L$ and $C_{r t d}$, where the resonating inductance is provided by the antenna. Indeed, $L$ could also be viewed as the inductance of the metal connection between the decoupling capacitor and the RTD device. Therefore, the equivalent circuit of the antenna load can be seen as the parallel of its inductance $L$ and resistance $R_{L}$, as shown in Fig. 8 b).

It is important to underline that, in Fig. 12, the diode is located at the centre of the slot. However, since the input impedance of the antenna is infinity at the centre and zero 


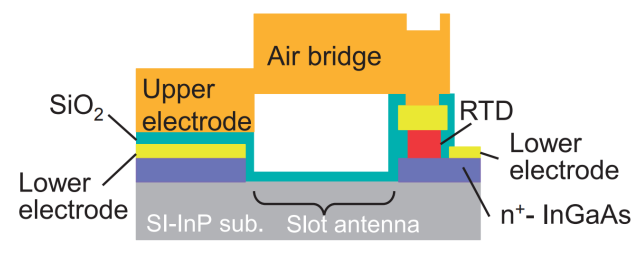

a)

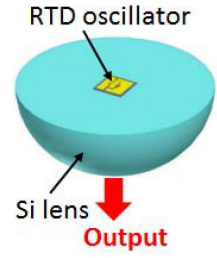

b)
Fig. 14. In a), a schematic of the $1.92 \mathrm{THz}$ oscillator layout cross section, showing the integration of the RTD device with the slot-antenna through the air-bridge structure (reprinted from [84] with permission). In b), a sketch showing the oscillator chip mounted onto the hemispherical Si lens for field collection and focusing (reprinted from [69] with permission).

at the edges of the slot, the RTD should be placed away from the centre for good impedance matching between the device and the antenna [135], where the exact location can be determined from 3D electromagnetic simulations. For this realisation, the dimension of the shorter part determines the antenna susceptance, which is mainly inductive and defines the oscillation frequency, while the dimensions of the longer part determine the radiation conductance, which determines the output power of the oscillator. Using this approach, RF powers of up to $\simeq 0.42 \mathrm{~mW}$ at a fundamental oscillation frequency of $548 \mathrm{GHz}$ have been demonstrated [136].

Furthermore, different oscillator cavity implementations have been proposed for frequency and RF power enhancement in the THz range, including circular [137], rectangular [138], and split ring [139], as well as simplified layouts without MIM capacitors [140]. In addition, mutual injection locking between two synchronised RTD oscillators has been demonstrated, which provided $\simeq 0.61 \mathrm{~mW}$ of $\mathrm{CW}$ radiation at a fundamental oscillation frequency of $620 \mathrm{GHz}$ [121]. Large scale arrays have also been proposed [127], featuring $\simeq 0.73 \mathrm{~mW}$ at $1 \mathrm{THz}$ in pulsed-mode, but the oscillators were not phaselocked. Further, a varactor diode integrated with the RTD was proposed to increase the frequency tunability range by overcoming the weak bias dependence of the device selfcapacitance, e.g. up to $\sim 40 \%$ [141].

The layout of the RTD THz slot-antenna oscillator operating at $1.92 \mathrm{THz}$ is depicted in Fig. 13. The upper RTD electrode was connected to the top antenna electrode along the back side of the slot through an air-bridge structure, as shown in Fig. 14 a), while the RTD bottom contact was connected to the bottom antenna electrode on the front side of the slot, integrating the RTD device with the antenna. The decoupling MIM capacitor was placed on the back side and it was fabricated through a thin $\mathrm{SiO}_{2}$ layer sandwiched between the upper and lower $\mathrm{Au}$ based antenna electrodes. The stabilising shunt resistor was realised from the heavily-doped InGaAs emitter contact layer and connected between the upper and bottom electrodes of the MIM capacitor and RTD device. The oscillator chip was mounted onto a Si lens, as shown in Fig. 14 b). The diode had a mesa area of $\sim 0.1 \mu \mathrm{m}^{2}$ and the measured output power was $0.4 \mu \mathrm{W}$ at $\simeq 1.92 \mathrm{THz}$ [84]. By further optimising the antenna electrode thickness $(3 \mu \mathrm{m})$ to reduce the associated conduction losses, fundamental oscillations of up to $\simeq 1.98 \mathrm{THz}$ with

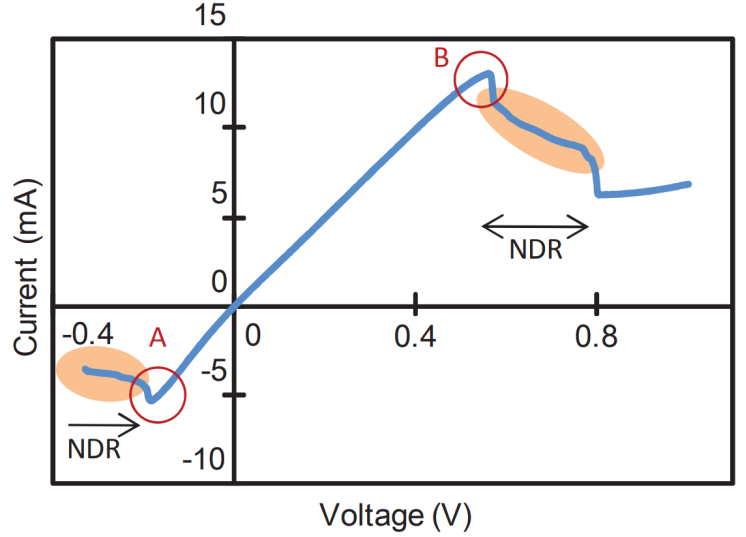

Fig. 15. Operation principle of a DBQW RTD detector based on the associated $I V$ curve. The device can be either biased close to the peak (marked as A and B depending on the polarisation region) in the case of a direct detector, as well as within the NDR region (highlighted in orange) if coherent detection is employed (reprinted from [146] with permission).

an output power of $40 \mathrm{nW}$ were achieved, employing a RTD with a mesa size of $\sim 0.2 \mu \mathrm{m}^{2}$ [38]. For the measurement of the oscillation frequency, a Fourier-transform infrared (FTIR) spectrometer with a liquid helium-cooled bolometer was used as a $\mathrm{THz}$ receiver while, for the output power measurement, the radiated $\mathrm{THz}$ wave was focused by a parabolic mirror and $\mathrm{THz}$ lenses, and then fed to a power meter via a horn-antenna. RTD THz oscillator concepts other than the aforementioned ones have also been reported in the literature. Their output powers are low and so are not discussed here in detail. For instance, they include a $675 \mathrm{GHz}$ differential double-RTD oscillator [128], and a $165 \mathrm{GHz}$ push-push [142] and 1.52 $\mathrm{THz}$ triple-push [126] oscillator based on second and third harmonics, respectively.

\section{RTD THZ DETECTORS}

Due to their superior speed performance, DBQW RTDs can be also used to realise high-sensitivity $\mathrm{THz}$ detectors [81] [82], and can operate both as standard direct [119], as well as coherent [143], detectors according to the specific bias point [144], as illustrated in Fig. 15. In the first case, the device is typically biased in close proximity to $V_{p}$ and can perform envelope detection of amplitude modulated signals (including OOK and ASK) according to the square-law scheme by exploiting the large associated $I V$ curve non-linearity [145] [146]. It is important to make it clear that, in contrast to SBDs, this non-linearity is not associated with thermionic emission, but rather with the thermal broadening of the QW subband associated with the ground-state resonant level, overcoming the thermal limit [52]. This makes the RT sensitivity of an RTD detector much higher than of a standard SBD, whose theoretical maximum DC current responsivity can go only up to $\sim 19.7 \mathrm{~A} / \mathrm{W}$ [147], while it is even lower for fieldeffect transistors (FETs) [148] [149]. For instance, III-V SBDs have shown voltage responsivities of up to $\simeq 1 \mathrm{kV} / \mathrm{W}$ at 250 $\mathrm{GHz}$ [150], while InP RTDs have demonstrated values of $\simeq$ $4 \mathrm{kV} / \mathrm{W}$ [151] and $\simeq 80 \mathrm{~V} / \mathrm{W}$ [152] at $0.35 \mathrm{THz}$ and 0.76 $\mathrm{THz}$, respectively, and current responsivities of up to $\simeq 7.3$ 


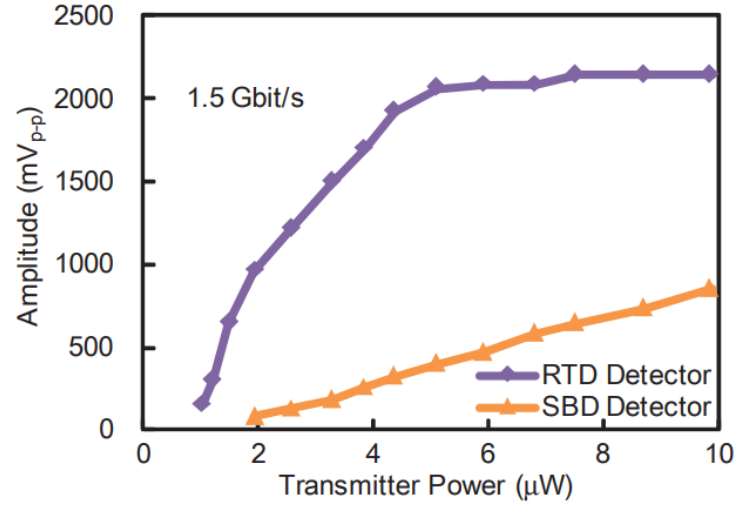

Fig. 16. Comparison of the measured output voltage versus Tx incoming $300 \mathrm{GHz} \mathrm{RF}$ power at $1.5 \mathrm{~Gb} / \mathrm{s}$ in the wireless experiment reported in [146] (reprinted from [146] with permission).

$\mathrm{A} / \mathrm{W}$ at $0.78 \mathrm{THz}$ [153]. Fig. 16 shows the measured output voltage versus the incoming $300 \mathrm{GHz}$ signal RF power of a wireless experiment where both an InP-based RTD and SBD direct detectors were employed for performance comparison, and shows a factor of up to 4 times higher sensitivity for the RTD detector [146]. The voltage responsivity of the RTD detector around $300 \mathrm{GHz}$ was up to $12 \mathrm{~dB}$ higher than the SBD one below the saturation voltage of the amplifier $(\sim 5$ $\mu \mathrm{W}$ ), and a maximum DC sensitivity of up to $30 \mathrm{~dB}$ higher was estimated from the $I V$ curve degree of non-linearity $\eta \propto d^{2} I(V) / d I(V)$ [147]. Voltage responsivities of up to $\sim 13 \mathrm{kV} / \mathrm{W}$ have been reported in the $26.5-50 \mathrm{GHz}$ band [154]. Note, however, that since the RTD is biased close to the NDR region, the amplitude of the detected signal must be reasonably small to avoid triggering oscillations due to the RTD instability, which would distort the incoming waveform, resulting in a lower dynamic range than SBDs [52].

Triple-barrier (TB) RTDs have also been proposed as highsensitivity $\mathrm{THz}$ direct detectors due to their superior prospective performance if compared with DBQW-based counterparts. The advantage lies in the strong current blocking due to the misalignment of the ground resonant subbands in the two adjacent QWs. When bias is applied and the resonant condition is met, the strong current increase leads to a strong non-linearity, with associated responsivity that can exceed the thermal limit [52]. TB RTDs can be used as high-sensitivity zero-bias detectors [155], with demonstrated voltage responsivities of up to $66 \mathrm{kV} / \mathrm{W}$ at $280 \mathrm{GHz}$ [156]. This exceptional performance of TB RTDs has not yet been exploited in system applications. TB RTDs have also been employed in THz sources [157][159], though their RF output powers tend to be low and so they are not discussed here.

Although direct detection can provide a simple solution for $\mathrm{THz}$ Rx systems, it relies only on the incoming amplitude information of the signal, which reduces spectral efficiency and sensitivity, limiting transmission distance and/or the corresponding data rate according to the specific link budget [145]. This apparent disadvantage can nonetheless be offset by the large available bandwidth at $\mathrm{THz}$ frequencies.

In the second type of RTD detector, the RTD device is embed-

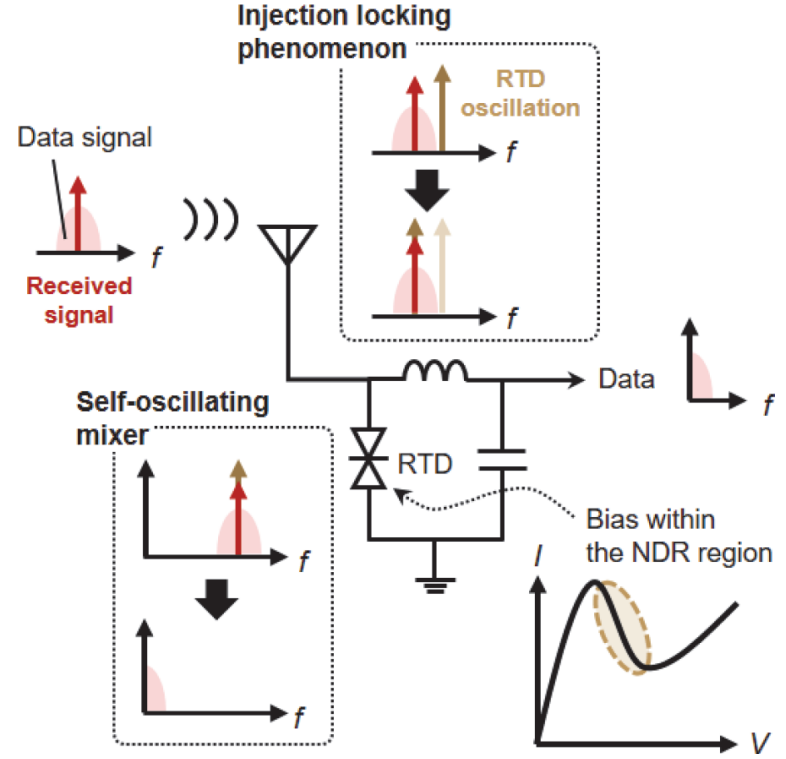

Fig. 17. Schematic illustration of the operation principle of an RTD coherent detector (reprinted from [143] with permission).

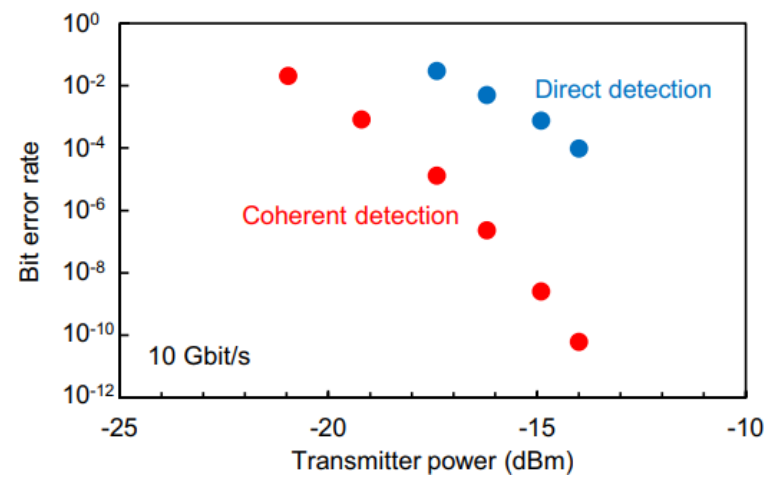

Fig. 18. Comparison of the measured BER versus Tx incoming RF power at $10 \mathrm{~Gb} / \mathrm{s}$ in the wireless experiment reported in [163], where the RTD bias point was changed to switch from direct to coherent detection (reprinted from [163] with permission)

ded in an oscillator circuit and operates as a coherent detector [144]. The principle of operation of this detector is illustrated in Fig. 17. In this case, the RTD is biased within its NDR region and the circuit acts as a local oscillator (LO) [160]. If the incoming carrier frequency $f_{c}$ is close enough to the LO one, injection locking [161] takes place and the two signals synchronise [162], performing coherent homodyne detection [143]. At the same time, the incoming signal is demodulated through the non-linear mixing properties of the NDR region [163], where the RTD acts as a self-oscillating mixer [145]. Since the RTD works, at the same time, as a LO and mixer, this approach enables the realization of ultra-compact and highsensitivity Rx chips. For instance, a sensitivity enhancement of up to $20 \mathrm{~dB}$ higher than direct detection was demonstrated in the wireless experiment reported in [163] employing a 300 GHz-band InP-based all-RTD TRx. In another experiment, a minimum noise equivalent power (NEP) of $\simeq 7.7 \mathrm{pW} / \sqrt{ } \mathrm{Hz}$ was reported at $0.78 \mathrm{THz}$ [144].

Together with the higher sensitivity due to the gain provided 
TABLE IV

THZ RTD WIRELESS COMMUNICATION LINKS SPECIFICS

\begin{tabular}{|c|c|c|c|c|c|c|c|}
\hline Ref. & $\mathrm{Tx}$ & $\mathrm{Rx}$ & $f_{c}[\mathrm{GHz}]$ & Modulation & Distance $[\mathrm{cm}]$ & Data rate $[\mathrm{Gb} / \mathrm{s}]$ & BER \\
\hline \multirow[t]{2}{*}{ [167] } & RTD & SBD & 490 & ASK & $20-30$ & 22 & $\mathrm{EF}$ \\
\hline & RTD & SBD & 490 & ASK & $20-30$ & 34 & $1.9 \times 10^{-3}$ \\
\hline \multirow[t]{2}{*}[168]{} & RTD & SBD & 650 & ASK & $20-30$ & 25 & $\mathrm{EF}$ \\
\hline & RTD & SBD & 650 & ASK & $20-30$ & 44 & $5 \times 10^{-4}$ \\
\hline \multirow{2}{*}{ [166] } & RTD & SBD & 62.5 & OOK & 30 & 10 & $\mathrm{EF}$ \\
\hline & RTD & SBD & 62.5 & OOK & 150 & 15 & $10^{-5}$ \\
\hline [169] & UTC-PD & RTD & 297 & OOK & 3 & 17 & $\mathrm{EF}$ \\
\hline \multirow{2}{*}{ [143] } & UTC-PD & RTD & 322 & OOK & 2 & 27 & EF \\
\hline & UTC-PD & RTD & 322 & OOK & 2 & 32 & $2.7 \times 10^{-2}$ \\
\hline \multirow[t]{2}{*}{ [151] } & UTC-PD & RTD & 350 & OOK & 3 & 32 & $\mathrm{EF}$ \\
\hline & UTC-PD & RTD & 350 & $\mathrm{OOK}$ & 3 & 36 & $9 \times 10^{-2}$ \\
\hline \multirow[t]{2}{*}{ [119] } & RTD & RTD & 286 & OOK & 10 & 9 & $\mathrm{EF}$ \\
\hline & RTD & RTD & 286 & OOK & 10 & 12 & $4 \times 10^{-3}$ \\
\hline \multirow[t]{2}{*}{ [176] } & RTD & RTD & 345 & OOK & 7.5 & 13 & $\mathrm{EF}$ \\
\hline & RTD & RTD & 345 & OOK & 7.5 & 20 & $2.1 \times 10^{-3}$ \\
\hline \multirow[t]{2}{*}{ [163] } & RTD & RTD & 343 & ASK & 7 & 30 & $\mathrm{EF}$ \\
\hline & RTD & RTD & 343 & ASK & 7 & 56 & $1.39 \times 10^{-5}$ \\
\hline [170] & RTD & SBD & $500-800$ & ASK & 20 & $56^{[a]}$ & $\sim 10^{-4}-10^{-3}$ \\
\hline [172] & UTC-PD & RTD & 324 & 16-QAM & 1 & 60 & $2 \times 10^{-3}$ \\
\hline [92] & RTD & SBD & 84 & ASK & 50 & 15 & $4.1 \times 10^{-3}$ \\
\hline \multirow[t]{2}{*}{ [165] } & RTD & SBD & 278 & ASK & 80 & 12 & $\mathrm{EF}$ \\
\hline & RTD & SBD & 278 & ASK & 80 & 22 & $10^{-3}$ \\
\hline [83] & RTD & SBD & 260 & ASK & 1 & 13 & - \\
\hline [173] & UTC-PD & RTD & $324-335$ & OOK & $2-3$ & $48^{[a]}$ & $\mathrm{EF}$ \\
\hline \multirow[t]{2}{*}{ [174] } & RTD & RTD & 354 & OOK & 7 & 25 & $\mathrm{EF}$ \\
\hline & RTD & RTD & 354 & OOK & 7 & 30 & $2 \times 10^{-3}$ \\
\hline
\end{tabular}

$[a]$ : multi-channel link.

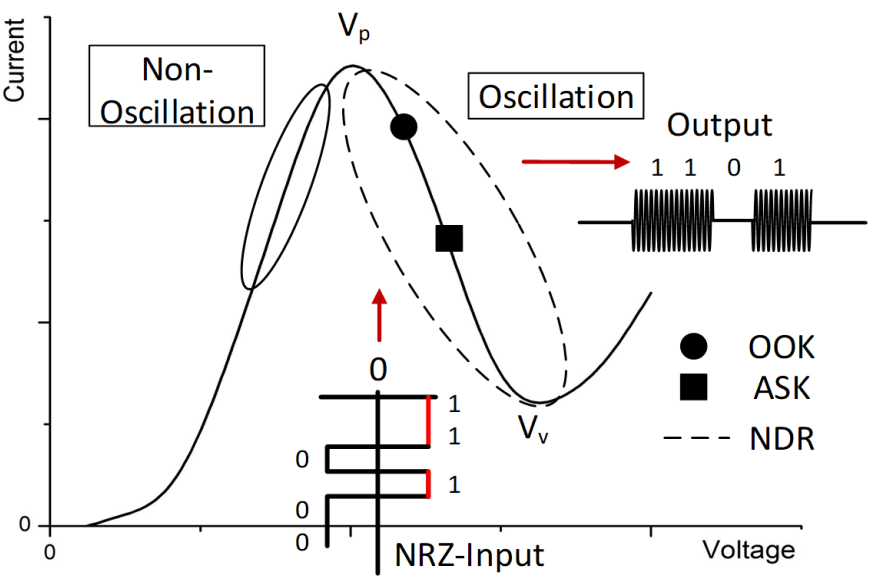

Fig. 19. Illustration of ASK/OOK modulation in RTD THz Tx (reprinted from [92] with permission).

by the device NDR, better spectral efficiency is possible, where the phase, frequency, and polarisation information of the received signal can be retrieved through injection locking [145]. Despite all the positive attributes, however, the use of a coherent RTD Rx is still very new and appropriate design

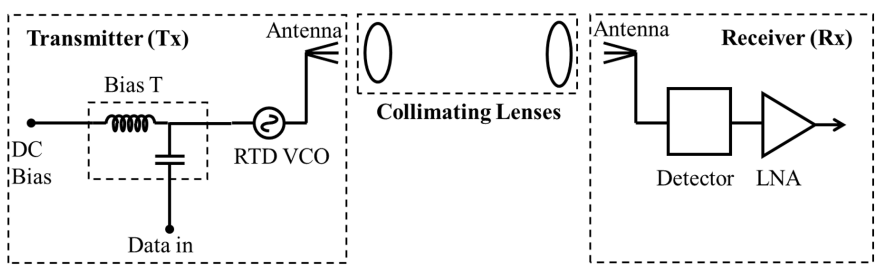

Fig. 20. Block diagram of a $\mathrm{THz}$ wireless system architecture employing an RTD-based Tx

methodologies to guarantee injection locking and maximise sensitivity need to be developed.

\section{RTD THZ WIRELESS COMMUNICATIONS}

In this section, state-of-the-art $\mathrm{THz}$ wireless communications employing RTDs are described. In these experiments, an RTD THz oscillator is usually employed at the Tx side. Both ASK and OOK modulation are applicable to the Tx depending on the bias level and the amplitude of data, as illustrated in Fig. 19. ASK modulation has been widely used because of the advantages of being a simple, low cost, high-bandwidth, and high-efficiency technique, while OOK, as a special case of ASK, implements data modulation by switching the carrier 


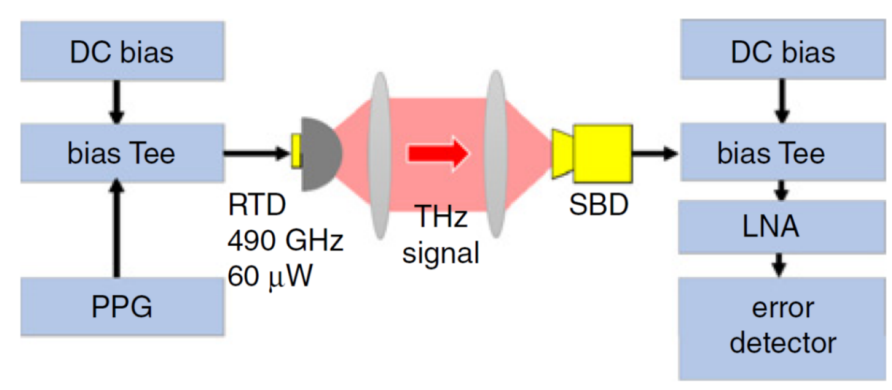

Fig. 21. Block diagram of the $\mathrm{THz}$ wireless data transmission experimental setup employed in [167] (reprinted from [167] with permission).
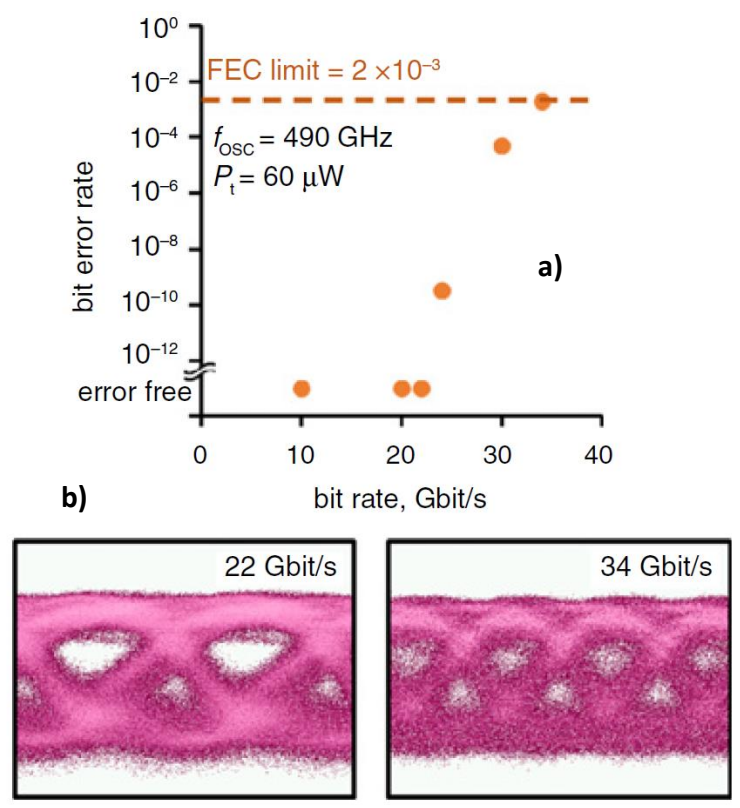

Fig. 22. Results of the wireless communication experiment reported in [167] In a), the measured BER as a function of the data rate, showing EF data transmission up to $22 \mathrm{~Gb} / \mathrm{s}$ and data rates of up to $34 \mathrm{~Gb} / \mathrm{s}$ with $\mathrm{BER} \simeq$ $1.9 \times 10^{-3}$, in $\mathrm{b}$ ), the corresponding measured eye diagrams (reprinted from [167] with permission).

on and off [145]. For ASK modulation, the RTD device is biased within its NDR region, while its bias point is set close to $V_{p}$ for input non-return to zero (NRZ) data in the case of OOK, in order to switch on and off the oscillator. Clearly, the input data amplitude for ASK is limited by the NDR voltage span, which is low. Since modulation of RTDs is via the bias line, the modulation bandwidth is mainly determined by the DC decoupling circuit comprising $C_{d c}$ and $R_{s t}$, as shown in Fig. 8 a), and has been measured to be around $110 \mathrm{GHz}$ for $300 \mathrm{GHz}$ oscillators [164].

\section{A. Wireless systems architecture}

The block diagram of a $\mathrm{THz}$ wireless link employing a RTD-based Tx is illustrated in Fig. 20. The Tx consists of an RTD voltage-controlled oscillator (VCO) and an external antenna or integrated on-chip antenna mounted on a hemispherical Si lens. THz lenses are often employed between the Tx and Rx antennas to focus and collimate the beam. The data is superimposed over the DC bias through a bias$\mathrm{T}$. Commercial power amplifiers are not yet available at $\mathrm{THz}$

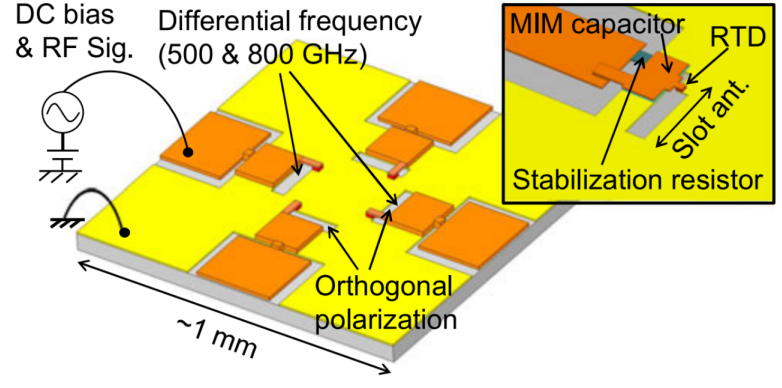

Fig. 23. Schematic representation of the RTD THz oscillator circuit layout employed at the Tx side for wireless data transmission through FDM and PDM (reprinted from [170] with permission).

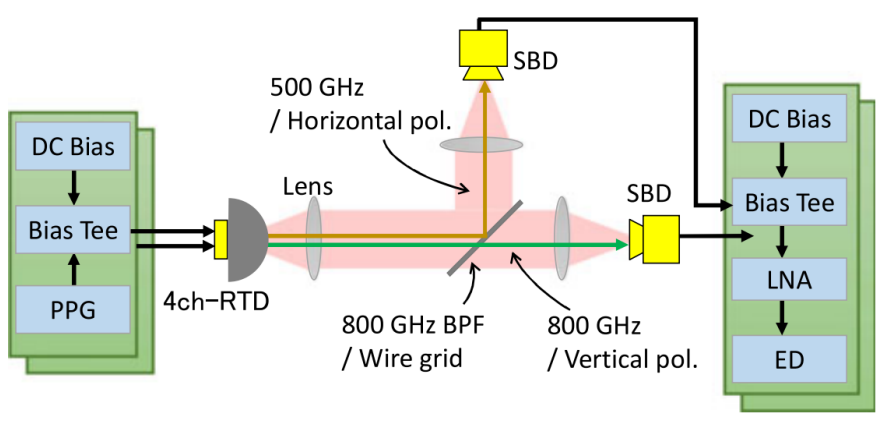

Fig. 24. Block diagram of the wireless communication experimental setup employed to perform data transmission through FDM and PDM (reprinted from [170] with permission).

frequencies and therefore none is employed at the Tx. At the Rx side, an antenna, which may be external or integrated, is also employed. The received signal is demodulated using either square-law-based direct detection, by employing an SBD or an RTD direct detector, or employing a coherent RTD detector. The received signal is amplified by a low noise amplifier (LNA). Table IV summarises some of the most recent wireless communication link results reported in the literature involving RTDs, either employed as a Tx, Rx, or both. In many cases, an SBD detector is used at the Rx while, in some other cases, an UTC-PD is employed at the Tx. The longest link distances of $50 \mathrm{~cm}$ [92], $80 \mathrm{~cm} \mathrm{[165],} \mathrm{and} 150 \mathrm{~cm} \mathrm{[166]} \mathrm{feature}$ Tx operating below $0.3 \mathrm{THz}$. For higher carrier frequencies $f_{c}$, link distances are under $30 \mathrm{~cm}$. As it is possible to notice, there is a clear correlation between the link distance and the Tx output power, which is in the milliwatt $(\mathrm{mW})$ range for the sub-100 $\mathrm{GHz} \mathrm{Tx}$ and microwatt $(\mu \mathrm{W})$ range for the ones operating at higher frequencies. Data rate results in the 9-60 $\mathrm{Gb} / \mathrm{s}$ range have been demonstrated, including a $30 \mathrm{~Gb} / \mathrm{s}$ error free (EF) transmission over a $7 \mathrm{~cm}$ long link through an allRTD TRx [163].

\section{B. Wireless data transmission}

Here, we describe some of the wireless transmission experiments in detail, the first in which a $490 \mathrm{GHz}$ oscillator was used as a Tx [167]. Direct modulation was carried out by superimposing a modulation signal onto the bias voltage through ASK. The experimental setup is shown in Fig. 21, where a pulse pattern generator (PPG) was used to impress 
a)
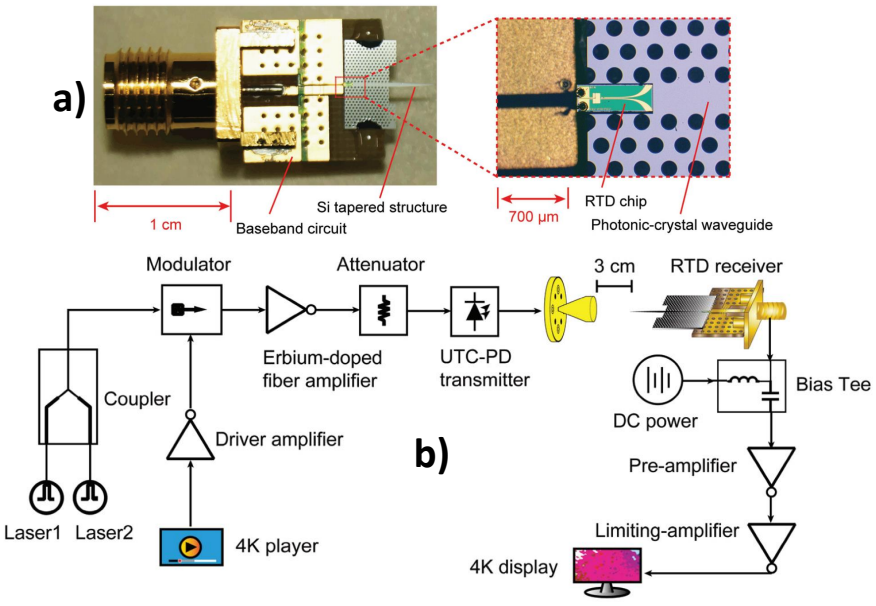

Fig. 25. In a), a photograph of the direct RTD-based Rx front-end module reported in [151], with a zoom in of the RTD integrated with a Si-based photonic crystal waveguide through a tapered-slot mode coupler, in b), the wireless experimental setup used for real-time high-resolution $4 \mathrm{~K}$ video transmission (reprinted from [151] with permission)
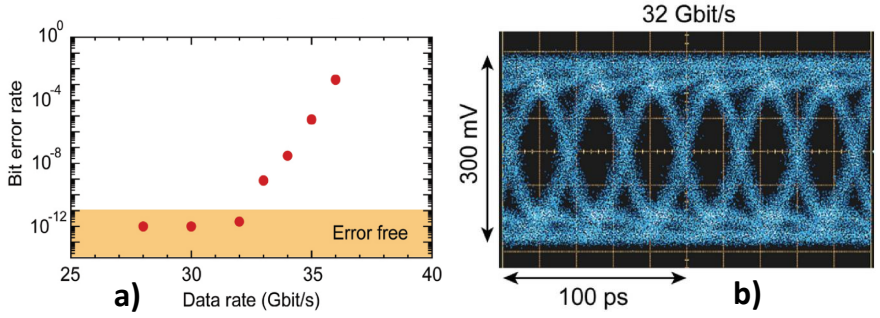

Fig. 26. Results of the wireless communication experiment reported in [151]. In a), the BER as a function of the data rate, in b), the eye diagram at 32 $\mathrm{Gb} / \mathrm{s}$ (reprinted from [151] with permission).

digital data over the carrier. The modulated $\mathrm{THz}$ signal was received through a horn-antenna, demodulated by an SBD direct detector and amplified through a LNA. The link distance between the Tx and the Rx was set to $20-30 \mathrm{~cm}$. The demodulated signal was then measured through an error detector (ED) and an oscilloscope. The results are shown in Fig. 22. Clear eye opening and EF data transmission were obtained at a data rate of $\simeq 22 \mathrm{~Gb} / \mathrm{s}$, while data rates of up to $\simeq 34 \mathrm{~Gb} / \mathrm{s}$ where achieved with $\mathrm{BER} \simeq 1.9 \times 10^{-3}$.

Recently, multi-channel wireless data transmission employing polarisation and frequency division multiplexing (PDM/FDM) schemes have also been reported [170]. Fig. 23 shows a schematic representation of the Tx chip circuit layout. Four slot-antenna RTD oscillators were integrated together: two of them oscillated at $500 \mathrm{GHz}$ and the other two at 800 $\mathrm{GHz}$ for FDM, while the two oscillators, at each of these two frequencies, had polarizations that were orthogonal to each other, realised through the relative orientation of their antennas layouts (perpendicular to each other), for PDM. The wireless communication setup is depicted in Fig. 24, where SBD detectors were employed at the Rx front-ends, together with horn-antennas. Data rates of up to $\simeq 56 \mathrm{Gbps}(\simeq 28$ Gbps per channel) were obtained using FDM in the 500 $\mathrm{GHz}$ and $800 \mathrm{GHz}$ channels with $\mathrm{BER} \simeq 2.3 \times 10^{-4}$ and $\simeq 1.5 \times 10^{-3}$, respectively, and through $\mathrm{PDM}$ at $500 \mathrm{GHz}$

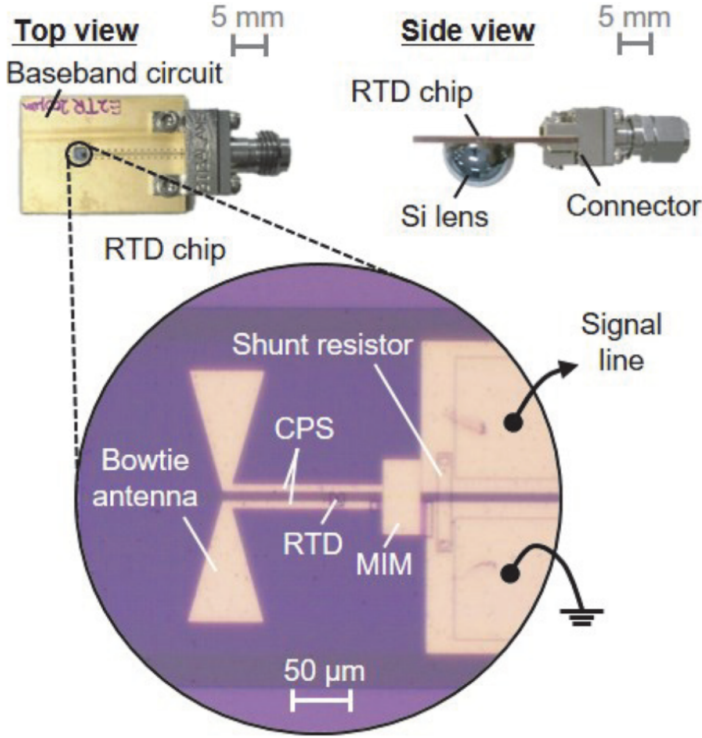

Fig. 27. Photographs of the $300 \mathrm{GHz}$-band RTD coherent Rx front-end module and a zoom of the RTD oscillator chip (reprinted from [143] with permission)

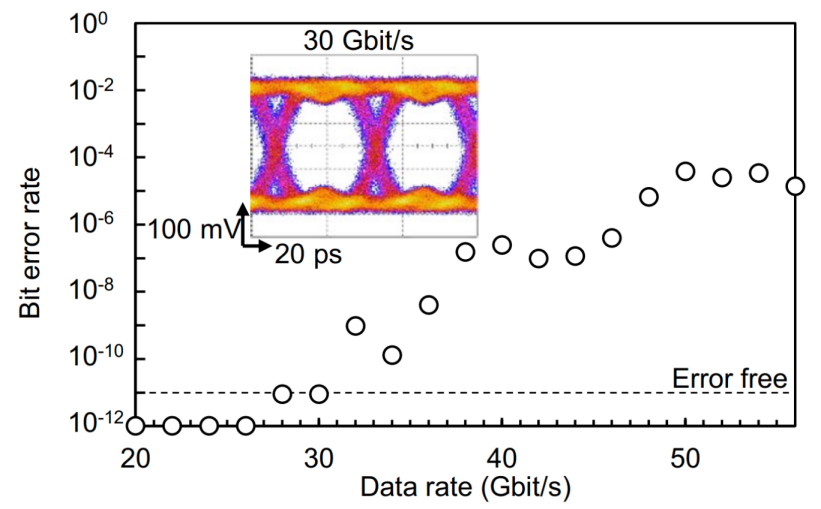

Fig. 28. Measured BER versus data rate of the wireless data transmission experiment reported in [163] (reprinted from [163] with permission).

with $\mathrm{BER} \simeq 1.5 \times 10^{-3}$ and $\simeq 1.4 \times 10^{-4}$ for the vertical and horizontal polarization channels, respectively.

Fig. 25 b) shows a $\mathrm{THz}$ wireless data transmission experimental setup involving an RTD as a direct detector [151]. The Tx was based on an UTC-PD-based photo-mixer, providing $f_{c} \sim$ $350 \mathrm{GHz}$, which was modulated through OOK. The detector was integrated with a Si-based photonic crystal waveguide platform [171] and had a measured voltage responsivity $\simeq 4$ kV/W. Fig. 25 a) shows a photograph of the $\mathrm{Rx}$ front-end module, outlining the metal-based tapered-slot mode converter and the photonic crystal waveguide. Fig. 26 shows the experimental results, where $\mathrm{EF}$ data transmission of up to $\simeq 32$ $\mathrm{Gb} / \mathrm{s}$ and data rates of up to $\simeq 36 \mathrm{~Gb} / \mathrm{s}$ with $\mathrm{BER} \simeq 9 \times 10^{-2}$ were achieved over $3 \mathrm{~cm}$ distance. Using a similar setup and 16-QAM, record date rates of up to $\simeq 60 \mathrm{~Gb} / \mathrm{s}$ with $\mathrm{BER}$ $\simeq 2 \times 10^{-3}$ over a $10 \mathrm{~mm}$ long wireless link were demonstrated at $\sim 324 \mathrm{GHz}$ [172]. In addition, multi-channel EF wireless data transmission through $\mathrm{OOK}$ of up to $\simeq 48 \mathrm{~Gb} / \mathrm{s}$ was demonstrated in the $300 \mathrm{GHz}$ band over a distance of $\sim 2-3$ 
cm using a UTC-PD-based Tx and an RTD direct Rx [173]. When a coherent RTD detector was employed, a coplanar stripline (CPS) was used to realise the resonator of the oscillator circuit and to connect the RTD device to a bow-tie antenna, while the Rx chip was mounted onto a Si lens to increase the antenna gain, as shown in Fig. 27. The experimental wireless communication setup was similar to the one shown in Fig. 25 b). The link distance was set to $2 \mathrm{~cm}$, while an oscilloscope and an ED were used to measure the demodulated signal. EF data transmission through $\mathrm{OOK}$ at around $322 \mathrm{GHz}$ of up to $\simeq 27$ $\mathrm{Gb} / \mathrm{s}$ and data rates of up to $\simeq 32 \mathrm{~Gb} / \mathrm{s}$ with $\mathrm{BER} \simeq 2.7 \times 10^{-2}$ were reported [143], with a sensitivity enhancement of around $10 \mathrm{~dB}$ with respect to the direct detection counterpart.

In the $\mathrm{THz}$ wireless data links reported in [119], [163], [175], and [176], an RTD was employed at both the Tx and Rx sides, by exploiting both the direct [119] [175] [176] as well as the coherent [163] detection scheme. With an allRTD wireless TRx setup and coherent detection, EF data transmission through ASK of up to $\simeq 30 \mathrm{~Gb} / \mathrm{s}$ and data rates of up to $\simeq 56 \mathrm{~Gb} / \mathrm{s}$ with $\mathrm{BER}$ of $\simeq 1.39 \times 10^{-5}$ were reported in the $300 \mathrm{GHz}$-band over a link distance of $7 \mathrm{~cm}$ [163]. A sensitivity enhancement of up to $40 \mathrm{~dB}$ with respect to the direct detection approach was demonstrated, where the associated measured BER versus data rate is shown in Fig. 28. On the other hand, using a direct detection [119], EF wireless data transmission through $\mathrm{OOK}$ of up to $\simeq 9 \mathrm{~Gb} / \mathrm{s}$ and record data rates of up to $\simeq 12 \mathrm{~Gb} / \mathrm{s}$ with $\mathrm{BER}$ of $\simeq 4 \times 10^{-3}$ were reported in the $300 \mathrm{GHz}$-band over a distance of $10 \mathrm{~cm}$. In the setup described in [175], data in the optical domain was converted into an electrical signal and used to modulate the RTD. OOK data was modulated using an intensity modulator and, after passing through a $1 \mathrm{~km}$ long fibre, it was converted to an electrical signal using a photo-diode (PD). The output signal was added to a DC biasing voltage using a bias- $\mathrm{T}$ and the resultant signal used to modulate the RTD at the Tx. Using multi-chip code division multiple access (CDMA), EF data rates of up to $\simeq 13 \mathrm{~Gb} / \mathrm{s}$ and of up to $\simeq 20 \mathrm{~Gb} / \mathrm{s}$ with BER of $\simeq 2.1 \times 10^{-3}$ were demonstrated [176].

Although data rates of several tens of $\mathrm{Gb} / \mathrm{s}$ have been achieved employing RTD devices at the Tx and/or Rx side, including single-channel EF data transmission at $30 \mathrm{~Gb} / \mathrm{s}$ and record data rates of up to $56 \mathrm{~Gb} / \mathrm{s}$ employing an all-RTD TRx, the link distance is still limited to the centimetre range at carrier frequencies above $\sim 300 \mathrm{GHz}$, while no wireless communication experiment has been reported at frequencies above $0.8 \mathrm{THz}$, which is inherently caused by the low output power of the Tx.

It would therefore seem that, in the $300 \mathrm{GHz}-b a n d$ and above, RTDs are more promising for practical $\mathrm{THz}$ wireless communication applications if compared to conventional transistor electronics for many reasons, including simplicity (a single RTD device can provide $>1 \mathrm{~mW}$ RF power compared to a transistor-based amplifier with typically 3-4 stages), low-cost lithography requirements (micron-sized RTD devices vs sub$100 \mathrm{~nm}$ gate transistors), and highly-sensitive RTD detectors (and so monolithic TRx can be realised). Moreover, the RTD can be epitaxially integrated with transistors, where the latter can provide basic functionalities, which could lead to the desired versatile $\mathrm{THz}$ systems.

\section{Challenges AND Future Perspectives}

RTD technology seems to offer the simplest and highest performance technology option for $\mathrm{THz}$ wireless TRx. The main weakness of the technology nowadays is the same for any $\mathrm{THz}$ technology, and it is represented by the low RF output power of the sources. As discussed throughout this paper, the main reasons for this includes underdeveloped device and circuit design techniques, in particular non-optimal epitaxial designs and circuit implementation approaches, and the lack of effective design techniques for arrays of RTD oscillators.

Current research trends suggest that increasing the RF power of RTD devices at $\mathrm{THz}$ frequencies is feasible and would enable the development of compact RTD $\mathrm{THz}$ ultra highspeed wireless TRx systems [163]. Individual sources with $\mathrm{mW}$-range output powers would facilitate the use of pulse amplitude modulation signalling. Such an approach would offer a way to dramatically reduce the Rx complexity, as no carrier synchronization is required. In that case, the total energy-per-bit will be significantly improved and will make the system more energy efficient, with expected efficiencies well below $10^{-1} \mathrm{pJ} / \mathrm{bit} / \mathrm{cm}$ [165].

Overcoming limitations associated with the high-permittivity InP substrate is another important challenge in RTD technology, since these have hindered the development of arrays of oscillators. Indeed, on-chip antennas have low gain, typically under $6 \mathrm{dBi}$, and the radiation is directed into the substrate. Therefore, the semiconductor dies are usually mounted on hemispherical lenses to collimate and focus the radiation, but these are bulky and make the systems cumbersome. As such, efforts to design sources with airside or upward radiation from the chips are underway. In this regard, on-chip radial-line slot-antenna (RLSA) arrays [123], together with dipole array [127] and patch [157] antenna configurations with dielectric supports for upward emission have been reported, though further innovations to this challenge are needed.

Also, because of the high-permittivity substrate, surface electromagnetic fields cannot be avoided, which cause unintentional coupling of arrays of oscillators and hinder proper synchronisation for spatial power combining. It thus remains a challenge to achieve mutual coupling with large scale arrays. Even though arrays of RTD oscillators have been reported, they have fallen short of delivering the expected high output power. RF powers of up to $\simeq 0.61 \mathrm{~mW}$ at $620 \mathrm{GHz}, \simeq 0.27$ $\mathrm{mW}$ at $770 \mathrm{GHz}$, and $\simeq 0.18 \mathrm{~mW}$ at $810 \mathrm{GHz}$ have been reported by employing a two-element frequency-locked oscillator array for CW coherent emission [121]. Most realisations, however, remain unsynchronised. For instance, a 16-element and a 64-element arrays provided output powers of up to $\simeq 28 \mu \mathrm{W}$ at a fundamental frequencies of $290 \mathrm{GHz}$ and 650 $\mathrm{GHz}$, respectively [131]. Earlier efforts in this regard included quasi-optical resonators for oscillators stabilisation [177] and power combining [178]. And recently, pulsed emission with RF powers of up to $\simeq 0.73 \mathrm{~mW}$ at around $1 \mathrm{THz}$ was reported by employing an unsynchronised 89-element large scale array [127], but the RF power have remained below the $1 \mathrm{~mW}$ level. 
Thus, new approaches for this challenge are required. At the device level, accurate characterisation and modelling of the RTD, especially of the key NDR region, is still nontrivial due to device instability. It would be advantageous to develop robust characterisation techniques which would enable the development of a complete physics-based non-linear largesignal model of the RTD device at $\mathrm{THz}$ frequencies in both NDR and PDR regions [52] [110]. The availability of such a model would enable the full non-linear dynamic analysis of the device in its entire operation frequency range. Moreover, quantum-transport-based numerical techniques aimed at accurately estimate the RTD DC $I V$ characteristic and capacitance are still required.

Even though nowadays THz RTD technology is based on InP, the future may belong to antimonides-based RTDs [57] [179]. Indeed, beyond around $300 \mathrm{GHz}$, the advantages of semiinsulating (SI) substrates, such InP, become less important in terms of insulation from the $\mathrm{THz}$ circuitry. In earlier efforts, antimonides-based RTDs were grown on either GaAs or gallium antimonide (GaSb) substrates, and so suffered from poor material quality due to the large lattice mismatch between the substrates and the device epitaxial layers [180] [181]. At $\mathrm{THz}$ frequencies, there is no apparent need for such substrates, since they can be electrically insulated from the passive $\mathrm{THz}$ circuitry, and so growth on the conductive but lattice-matched InAs substrates of high-quality InAs/AlSb RTDs can be undertaken. Compared with InP-based RTDs, antimonides-based RTDs promise superior speed and power performances [182], and so may underpin future THz TRx systems.

Beyond wireless communications, it is important to mention that THz RTD oscillators are also being developed for other applications, including compact high-resolution imaging [183]-[185], spectroscopy [186], and radar [187]-[189] systems. It would therefore seem that the RTD is a very promising candidate technology for many practical $\mathrm{THz}$ applications.

\section{ACKNOWLEDGMENTS}

The authors would like to thank the members, both past and present, of the High-Frequency Electronics group, division of Electronics and Nanoscale Engineering, James Watt School of Engineering, University of Glasgow, including Dr. Liquan Wang, Dr. Khalid Alharbi, Dr. Afesomeh Ofiare, Dr. Andrei Catalin Cornescu, and Dr. Razvan Morariu for their contributions to some of the results presented in this paper, and the James Watt Nanofabrication Centre (JWNC) staff, University of Glasgow, for the support during related microfabrication. They would also like to express a special thank to Prof. Safumi Suzuki and Prof. Masahiro Asada of the Tokyo University of Technology, and Prof. Tadao Nagatsuma, Prof. Masayuki Fujita, and Prof. Julian Webber of Osaka University for providing precious details and insight to their work on RTDs. In addition, thanks are also due to the partners of the Horizon European 2020 projects in which a significant part of our RTD-related work has been developed, including iBROW, TERAPOD, and TeraApps. In particular, we would like to thank Prof. José Figueiredo of University of Lisbon for many fruitful discussions on RTD technology. The work of Davide Cimbri was supported by TeraApps (Doctoral Training Network in Terahertz Technologies for Imaging, Radar and Communication Applications), which received funding from the European Union's Horizon 2020 research and innovation programme under Marie Skłodowska-Curie Innovative Training Network (ITN) grant agreement No. 765426.

\section{REFERENCES}

[1] M. Shafi, A. F. Molisch, P. J. Smith, T. Haustein, P. Zhu, P. De Silva, F. Tufvesson, A. Benjebbour, and G. Wunder, "5G: A Tutorial Overview of Standards, Trials, Challenges, Deployment, and Practice," IEEE Journal on Selected Areas in Communications, vol. 35, no. 6, pp. 1201-1221, 2017, doi: 10.1109/JSAC.2017.2692307.

[2] Z. Chen, X. Ma, B. Zhang, Y. Zhang, Z. Niu, N. Kuang, W. Chen, L. Li, and S. Li, "A survey on terahertz communications," China Communications, vol. 16, no. 2, pp. 1-35, 2019, doi: 10.12676/j.cc.2019.02.001.

[3] A. Al-Fuqaha, M. Guizani, M. Mohammadi, M. Aledhari, and M. Ayyash, "Internet of Things: A Survey on Enabling Technologies, Protocols, and Applications," IEEE Communications Surveys \& Tutorials, vol. 17, no. 4, pp. 2347-2376, 2015, doi: 10.1109/COMST.2015.2444095.

[4] S. Cherry, "Edholm's law of bandwidth," IEEE Spectrum, vol. 41, no. 7, pp. 58-60, 2004, doi: 10.1109/MSPEC.2004.1309810.

[5] R. Piesiewicz, T. Kleine-Ostmann, N. Krumbholz, D. Mittleman, M. Koch, J. Schoebel, and T. Kurner, "Short-Range Ultra-Broadband Terahertz Communications: Concepts and Perspectives," IEEE Antennas and Propagation Magazine, vol. 49, no. 6, pp. 24-39, 2007, doi: 10.1109/MAP.2007.4455844.

[6] J. Federici and L. Moeller, "Review of terahertz and subterahertz wireless communications," Journal of Applied Physics, vol. 107, no. 11, pp. 111101, 2010, doi: 10.1063/1.3386413.

[7] M. Koch, "Terahertz Communications: A 2020 vision," in Terahertz Frequency Detection and Identification of Materials and Objects, pp. 325338, Springer, 2007, doi: 10.1007/978-1-4020-6503-3_18.

[8] P. Smulders, "The road to $100 \mathrm{~Gb} / \mathrm{s}$ wireless and beyond: basic issues and key directions," IEEE Communications Magazine, vol. 51, no. 12, pp. 86-91, 2013, doi: 10.1109/MCOM.2013.6685762.

[9] T. Kürner and S. Priebe, "Towards THz Communications - Status in Research, Standardization and Regulation," Journal of Infrared, Millimeter, and Terahertz Waves, vol. 35, no. 1, pp. 53-62, 2014, doi: 10.1007/s10762-013-0014-3.

[10] P. Yang, Y. Xiao, M. Xiao, and S. Li, "6G Wireless Communications: Vision and Potential Techniques, Standardization and Regulation," IEEE Network, vol. 33, no. 4, pp. 70-75, 2019, doi: 10.1109/MNET.2019.1800418.

[11] T. Nagatsuma, "Terahertz technologies: present and future," IEICE Electronics Express, vol. 8, no. 14, pp. 1127-1142, 2011, doi: 10.1587/elex.8.1127.

[12] H. -J. Song and T. Nagatsuma, "Present and Future of Terahertz Communications," IEEE Transactions on Terahertz Science and Technology, vol. 1, no. 1, pp. 256-263, 2011, doi: 10.1109/TTHZ.2011.2159552.

[13] H. -J. Song and N. Lee, "Terahertz Communications: Challenges in the Next Decade," IEEE Transactions on Terahertz Science and Technology, 2021, doi: 10.1109/TTHZ.2021.3128677.

[14] D. Pavlidis, "Fundamentals of Terahertz Devices and Applications," Wiley, 2021, doi: 10.1002/9781119460749.

[15] H. -J. Song, "Terahertz Wireless Communications: Recent Developments Including a Prototype System for Short-Range Data Downloading,' IEEE Microwave Magazine, vol. 22, no. 5, pp. 88-99, 2021, doi: 10.1109/MMM.2021.3056935.

[16] T. Nagatsuma, G. Ducournau, and C. C. Renaud, "Advances in terahertz communications accelerated by photonics," Nature Photonics, vol. 10, no. 6, pp. 371-379, 2016, doi: 10.1038/nphoton.2016.65.

[17] K. Sengupta, T. Nagatsuma, and D. M. Mittleman, "Terahertz integrated electronic and hybrid electronic-photonic systems," Nature Electronics, vol. 1, no. 12, pp. 622-635, 2018, doi: 10.1038/s41928-018-0173-2.

[18] S. Koenig, D. Lopez-Diaz, J. Antes, F. Boes, R. Henneberger, A. Leuther, A. Tessmann, R. Schmogrow, D. Hillerkuss, R. Palmer, T. Zwick, C. Koos, W. Freude, O. Ambacher, J. Leuthold, and I. Kallfass, "Wireless Sub-THz Communication System with High Data Rate," Nature Photonics, vol. 7, no. 12, pp. 977-981, 2013, doi: 10.1038/nphoton.2013.275. 
[19] T. Nagatsuma, K. Oogimoto, Y. Yasuda, Y. Fujita, Y. Inubushi, S Hisatake, A. M. Agoues, and G. C. Lopez, "300-GHz-Band Wireless Transmission at $50 \mathrm{Gbit} / \mathrm{s}$ over 100 Meters," 41st International Conference on Infrared, Millimeter, and Terahertz waves (IRMMW-THz), pp. 1-2, 2016, doi: 10.1109/IRMMW-THz.2016.7758356.

[20] T. Nagatsuma, Y. Fujita, Y. Yasuda, Y. Kanai, S. Hisatake, M. Fujiwara, and J. Kani, "Real-time 100-Gbit/s QPSK transmission using photonics-based 300-GHz-band wireless link," IEEE International Topical Meeting on Microwave Photonics (MWP), pp. 27-30, 2016, doi: 10.1109/MWP.2016.7791277.

[21] V. K. Chinni, P. Latzel, M. Zègaoui, C. Coinon, X. Wallart, E. Peytavit, J. F. Lampin, K. Engenhardt, P. Szriftgiser, M. Zaknoune, and G. Ducournau, "Single-channel $100 \mathrm{Gbit} / \mathrm{s}$ transmission using III-V UTC-PDs for future IEEE 802.15.3d wireless links in the $300 \mathrm{GHz}$ band," Electronics Letters, vol. 54, no. 10, pp. 638-640, 2018, doi: 10.1049/el.2018.0905.

[22] S. Jia, X. Pang, O. Ozolins, X. Yu, H. Hu, J. Yu, P. Guan, F. Da Ros, S. Popov, G. Jacobsen, M. Galili, T. Morioka, D. Zibar, and L. K. Oxenløwe, "0.4 THz Photonic-Wireless Link With 106 Gb/s Single Channel Bitrate," Journal of Lightwave Technology, vol. 36, no. 2, pp. 610-615, 2018, doi: 10.1109/JLT.2017.2776320.

[23] X. Yu, S. Jia, H. Hu, M. Galili, T. Morioka, P. U. Jepsen, and L. K. Oxenløwe, "160 Gbit/s photonics wireless transmission in the 300$500 \mathrm{GHz}$ band," APL Photonics, vol. 1, no. 8, pp. 081301, 2016, doi: $10.1063 / 1.4960136$

[24] T. Ishibashi, Y. Muramoto, T. Yoshimatsu, and H. Ito, "UnitravelingCarrier Photodiodes for Terahertz Applications," IEEE Journal of Selected Topics in Quantum Electronics, vol. 20, no. 6, pp. 79-88, 2014, doi: 10.1109/JSTQE.2014.2336537.

[25] S. Preu, G. H. Döhler, S. Malzer, L. J. Wang, and A. C. Gossard "Tunable, continuous-wave Terahertz photomixer sources and applications," Journal of Applied Physics, vol. 109, no. 6, pp. 061301, 2011, doi: 10.1063/1.3552291.

[26] J. Nishizawa, P. Płotka, T. Kurabayashi, and H. Makabe, "706-GHz GaAs CW fundamental-mode TUNNETT diodes fabricated with molecular layer epitaxy," physica status solidi (c), vol. 5, no. 9, pp. 2802-2804, 2008, doi: 10.1002/pssc.200779256.

[27] A. Acharyya and J. P. Banerjee, "Prospects of IMPATT devices based on wide bandgap semiconductors as potential terahertz sources," Applied Nanoscience, vol. 4, no. 1, pp. 1-14, 2014, doi: 10.1007/s13204-0120172-y.

[28] H. Eisele, "480 GHz oscillator with an InP Gunn device," Electronics Letters, vol. 46, no. 6, pp. 422-423, 2010, doi: 10.1049/el.2010.3362.

[29] A. Khalid, G. M. Dunn, R. F. Macpherson, S. Thoms, D. Macintyre, C. Li, M. J. Steer, V. Papageorgiou, I. G. Thayne, M. Kuball, C. H. Oxley, M. Montes Bajo, A. Stephen, J. Glover, and D. R. S. Cumming, "Terahertz oscillations in an $\operatorname{In}_{0.53} \mathrm{Ga}_{0.47}$ As submicron planar Gunn diode," Journal of Applied Physics, vol. 115, no. 11, pp. 114502, 2014, doi: 10.1063/1.4868705.

[30] I. Mehdi, J. V. Siles, C. Lee, and E. Schlecht, "THz Diode Technology: Status, Prospects, and Applications," Proceedings of the IEEE, vol. 105, no. 6, pp. 990-1007, 2017, doi: 10.1109/JPROC.2017.2650235.

[31] H. Eisele, L. Li, and E. H. Linfield, "High-performance GaAs/AlAs superlattice electronic devices in oscillators at frequencies 100-320 GHz," Applied Physics Letters, vol. 112, no. 17, pp. 172103, 2018, doi: 10.1063/1.5020265.

[32] U. R. Pfeiffer, R. Jain, J. Grzyb, S. Malz, P. Hillger, and P. RodríguezVízquez, "Current Status of Terahertz Integrated Circuits - From Components to Systems," IEEE BiCMOS and Compound Semiconductor Integrated Circuits and Technology Symposium (BCICTS), pp. 1-7, 2018, doi: 10.1109/BCICTS.2018.8551068.

[33] M. Asada and S. Suzuki, "Terahertz Emitter Using Resonant-Tunneling Diode and Applications," Sensors, vol. 21, no. 4, pp. 1384, 2021, doi: $10.3390 / \mathrm{s} 21041384$.

[34] H. -J. Lee, S. Rami, S. Ravikumar, V. Neeli, K. Phoa, B. Sell, and Y. Zhang, "Intel 22nm FinFET (22FFL) Process Technology for RF and $\mathrm{mm}$ Wave Applications and Circuit Design Optimization for FinFET Technology,' IEEE International Electron Devices Meeting (IEDM), pp. 14.1.1-14.1.4, 2018, doi: 10.1109/IEDM.2018.8614490.

[35] B. Heinemann, H. Rücker, R. Barth, F. Bärwolf, J. Drews, G. G Fischer, A. Fox, O. Fursenko, T. Grabolla, F. Herzel, J. Katzer, J. Korn, A. Krüger, P. Kulse, T. Lenke, M. Lisker, S. Marschmeyer, A. Scheit, D. Schmidt, J. Schmidt, M. A. Schubert, A. Trusch, C. Wipf, and D. Wolansky, "SiGe HBT with $f_{x} / f_{\max }$ of $505 \mathrm{GHz} / 720 \mathrm{GHz}$," IEEE International Electron Devices Meeting (IEDM), pp. 3.1.1-3.1.4, 2016, doi: 10.1109/IEDM.2016.7838335.

[36] M. Urteaga, R. Pierson, P. Rowell, V. Jain, E. Lobisser, and M. J. W. Rodwell, "130nm InP DHBTs with $\mathrm{ft}>0.52 \mathrm{THz}$ and fmax
$>$ 1.1THz," 69th Device Research Conference, pp. 281-282, 2011, doi: 10.1109/DRC.2011.5994532.

[37] X. Mei, W. Yoshida, M. Lange, J. Lee, J. Zhou, P. Liu, K. Leong, A. Zamora, J. Padilla, S. Sarkozy, R. Lai, and W. R. Deal, "Firs Demonstration of Amplification at $1 \mathrm{THz}$ Using 25-nm InP High Electron Mobility Transistor Process," IEEE Electron Device Letters, vol. 36, no. 4, pp. 327-329, 20115, doi: 10.1109/LED.2015.2407193.

[38] R. Izumi, S. Suzuki, and M. Asada, "1.98 THz resonant-tunneling-diode oscillator with reduced conduction loss by thick antenna electrode," 42nd International Conference on Infrared, Millimeter, and Terahertz Waves (IRMMW-THz), pp. 1-2, 2017, doi: 10.1109/IRMMW-THz.2017.8066877.

[39] T. Nagatsuma, S. Hisatake, M. Fujita, H. H. N. Pham, K. Tsuruda, S. Kuwano, and J. Terada, "Millimeter-Wave and Terahertz-Wave Applications Enabled by Photonics," IEEE Journal of Quantum Electronics, vol 52, no. 1, pp. 1-12, 2016, doi: 10.1109/JQE.2015.2506992.

[40] D. Kim and S. Jeon, "A 300-GHz High-Power High-Efficiency VoltageControlled Oscillator With Low Power Variation," IEEE Microwave and Wireless Components Letters, vol. 30, no. 5, pp. 496-499, 2020, doi: 10.1109/LMWC.2020.2986160.

[41] P. Hillger, J. Grzyb, S. Malz, B. Heinemann, and U. Pfeiffer, "A lensintegrated $430 \mathrm{GHz}$ SiGe HBT source with up to $-6.3 \mathrm{dBm}$ radiated power," IEEE Radio Frequency Integrated Circuits Symposium (RFIC), pp. 160-163, 2017, doi: 10.1109/RFIC.2017.7969042.

[42] W. Steyaert and P. Reynaert, "A $0.54 \mathrm{THz}$ Signal Generator in $40 \mathrm{~nm}$ Bulk CMOS With $22 \mathrm{GHz}$ Tuning Range and Integrated Planar Antenna," IEEE Journal of Solid-State Circuits, vol. 49, no. 7, pp. 1617-1626, 2014, doi: 10.1109/JSSC.2014.2319251.

[43] K. M. K. H. Leong, X. Mei, W. H. Yoshida, A. Zamora, J. G. Padilla, B. S. Gorospe, K. Nguyen, and W. R. Deal, "850 GHz Receiver and Transmitter Front-Ends Using InP HEMT," IEEE Transactions on Terahertz. Science and Technology, vol. 7, no. 4, pp. 466-475, 2017, doi: 10.1109/TTHZ.2017.2710632.

[44] U. R. Pfeiffer, Y. Zhao, J. Grzyb, R. Al Hadi, N. Sarmah, W. Förster, H. Rücker, and Bernd Heinemann, "A $0.53 \mathrm{THz}$ reconfigurable source array with up to $1 \mathrm{~mW}$ radiated power for terahertz imaging applications in $0.13 \mu \mathrm{m} \mathrm{SiGe} \mathrm{BiCMOS,"} \mathrm{IEEE} \mathrm{International} \mathrm{Solid-State} \mathrm{Circuits}$ Conference Digest of Technical Papers (ISSCC), pp. 256-257, 2014, doi: 10.1109/ISSCC.2014.6757424.

[45] R. Han, C. Jiang, A. Mostajeran, M. Emadi, H. Aghasi, H. Sherry, A. Cathelin, and E. Afshari, "A SiGe Terahertz Heterodyne Imaging Transmitter With $3.3 \mathrm{~mW}$ Radiated Power and Fully-Integrated PhaseLocked Loop,' IEEE Journal of Solid-State Circuits, vol. 50, no. 12, pp. 2935-2947, 2015, doi: 10.1109/JSSC.2015.2471847.

[46] E. Öjefors, J. Grzyb, Y. Zhao, B. Heinemann, B. Tillack, and U. R. Pfeiffer, "A $820 \mathrm{GHz}$ SiGe chipset for terahertz active imaging applications," IEEE International Solid-State Circuits Conference, pp. 224-226, 2011, doi: 10.1109/ISSCC.2011.5746294.

[47] Z. Hu, M. Kaynak and R. Han, "High-Power Radiation at $1 \mathrm{THz}$ in Silicon: A Fully Scalable Array Using a Multi-Functional Radiating Mesh Structure," IEEE Journal of Solid-State Circuits, vo. 53, no. 5, pp. 13131327, 2018, doi: 10.1109/JSSC.2017.2786682.

[48] K. Sengupta and A. Hajimiri, "A 0.28 THz Power-Generation and BeamSteering Array in CMOS Based on Distributed Active Radiators," IEEE Journal of Solid-State Circuits, vo. 47, no. 12, pp. 3013-3031, 2012, doi: 10.1109/JSSC.2012.2217831.

[49] I. Kallfass, F. Boes, T. Messinger, J. Antes, A. Inam, U. Lewark, A. Tessmann, and R. Henneberger, "64 Gbit/s Transmission over $850 \mathrm{~m}$ Fixed Wireless Link at $240 \mathrm{GHz}$ Carrier Frequency," Journal of Infrared, Millimeter, and Terahertz Waves, vo. 36, no. 2, pp. 221-233, 2015, doi 10.1007/s10762-014-0140-6.

[50] H. Mizuta and T. Tanoue, "The Physics and Applications of Resonant Tunnelling Diodes," Cambridge University Press, 1995, doi: 10.1017/CBO9780511629013.

[51] B. Ricco and M. Y. Azbel, "Physics of resonant tunneling. The onedimensional double-barrier case," Physical Review B, vol. 29, no. 4, pp. 1970-1981, 1984, doi: 10.1103/PhysRevB.29.1970.

[52] M. Feiginov, "Frequency Limitations of Resonant-Tunnelling Diodes in Sub-THz and THz Oscillators and Detectors," Journal of Infrared, Millimeter, and Terahertz Waves, vol. 40, no. 4, pp. 365-394, 2019, doi: 10.1007/s10762-019-00573-5.

[53] M. Asada, S. Suzuki, and T. Fukuma, "Measurements of temperature characteristics and estimation of terahertz negative differential conductance in resonant-tunneling-diode oscillators," AIP Advances, vol. 7, no. 11, pp. 115226, 2017, doi: 10.1063/1.5007093.

[54] C. Kim and A. Brandli, "High-Frequency High-Power Operation of Tunnel Diodes," IRE Transactions on Circuit Theory, vol. 8, no. 4, pp. 416-425, 1961, doi: 10.1109/TCT.1961.1086849. 
[55] N. Orihashi, S. Hattori, S. Suzuki, and M. Asada, ”Experimental and Theoretical Characteristics of Sub-Terahertz and Terahertz Oscillations of Resonant Tunneling Diodes Integrated with Slot Antennas," Japanese Journal of Applied Physics, vol. 44, no. 11, pp. 7809-7815, 2005, doi: 10.1143/jjap.44.7809.

[56] M. Feiginov, C. Sydlo, O. Cojocari, and P. Meissner, "Resonanttunneling-diode oscillators operating at frequencies above $1.1 \mathrm{THz}$," Applied Physics Letters, vol. 99, no. 23, pp. 233506, 2011, doi: 10.1063/1.3667191.

[57] E. R. Brown, J. R. Soderstrom, C. D. Parker, L. J. Mahoney, K. M. Molvar, and T. C. McGill, "Oscillations up to $712 \mathrm{GHz}$ in InAs/AlSb resonant tunnelling diodes," Applied Physics Letters, vol. 58, no. 20, pp. 2291-2293, 1991, doi: 10.1063/1.104902.

[58] E. R. Brown, T. C. L. G. Sollner, C. D. Parker, W. D. Goodhue, and C. L. Chen, "Oscillations up to $420 \mathrm{GHz}$ in GaAs/AlAs resonant tunneling diodes," Applied Physics Letters, vol. 55, no. 17, pp. 1777-1779, 1989, doi: $10.1063 / 1.102190$

[59] M. Levinshtein, S. Rumyantsev, and M. Shur, "Handbook Series on Semiconductor Parameters," Volume 1 and 2, World Scientific, 1996, doi: $10.1142 / 2046$

[60] G. Martin, A. Botchkarev, A. Rockett, and H. Morkoç, "Valence-band discontinuities of wurtzite $\mathrm{GaN}$, AlN, and $\mathrm{InN}$ heterojunctions measured by x-ray photoemission spectroscopy," Applied Physics Letters, vol. 68, no. 18, pp. 2541-2543, 1996, doi: 10.1063/1.116177.

[61] O. Madelung, "Semiconductors: Data Handbook," Springer, 2004, doi: 10.1007/978-3-642-18865-7.

[62] I. Vurgaftman, J. R. Meyer, and L. R. Ram-Mohan, "Band parameters for III-V compound semiconductors and their alloys," Journal of Applied Physics, vol. 89, no. 11, pp. 5815-5875, 2001, doi: 10.1063/1.1368156.

[63] H. Sugiyama, H. Yokoyama, A. Teranishi, S. Suzuki, and M. Asada "Extremely High Peak Current Densities of over $1 \times 10^{6} \mathrm{~A} / \mathrm{cm}^{2}$ in InP-Based InGaAs/AlAs Resonant Tunneling Diodes Grown by MetalOrganic Vapor-Phase Epitaxy," Japanese Journal of Applied Physics, vol. 49, no. 5R, pp. 051201, 2010, doi: 10.1143/jjap.49.051201.

[64] R. Quay, C. Moglestue, V. Palankovski, and S. Selberherr, "A temperature dependent model for the saturation velocity in semiconductor materials," Materials Science in Semiconductor Processing, vol. 3, no. 1-2, pp. 149-155, 2000, doi: 10.1016/S1369-8001(00)00015-9.

[65] S. Masudy-Panah, Y. Wu, D. Lei, A. Kumar, Y. -C. Yeo, and X. Gong, "Nanoscale metal-InGaAs contacts with ultra-low specific contact resistivity: Improved interfacial quality and extraction methodology," Journal of Applied Physics, vol. 123, no. 2, pp. 024508, 2018, doi: 10.1063/1.4997686

[66] W. D. Zhang, T. A. Growden, E. R. Brown, P. R. Berger, D. F. Storm, and D. J. Meyer, "Fabrication and Characterization of GaN/AlN Resonant Tunneling Diodes," in High-Frequency GaN Electronic Devices, pp. 249281, Springer, 2020, doi: 10.1007/978-3-030-20208-8_9.

[67] J. Encomendero, R. Yan, A. Verma, S. M. Islam, V. Protasenko, S. Rouvimov, P. Fay, D. Jena, and H. G. Xing, "Room temperature microwave oscillations in GaN/AlN resonant tunneling diodes with peak current densities up to $220 \mathrm{kA} / \mathrm{cm}^{2}$," Applied Physics Letters, vol. 112, no. 10 , pp. 103101, 2018, doi: 10.1063/1.5016414.

[68] H. P. Zhang, J. S. Xue, Z. P. Sun, L. X. Li, J. J. Yao, F. Liu, X. Y. Yang, G. L. Wu, Z. M. Li, Y. R. Fu, Z. H. Liu, J. C. Zhang, and Y. Hao, "1039 $\mathrm{kA} / \mathrm{cm}^{2}$ peak tunneling current density in GaN-based resonant tunneling diode with a peak-to-valley current ratio of 1.23 at room temperature on sapphire substrate," Applied Physics Letters, vol. 119, no. 15, pp. 153506, 2021, doi: 10.1063/5.0064790.

[69] S. Suzuki and M. Asada, "Room-Temperature Oscillation of Resonan Tunneling Diodes close to $2 \mathrm{THz}$ and Their Functions for Various Applications," Journal of Infrared, Millimeter, and Terahertz Waves, vol. 37, no. 12, pp. 1185-1198, 2016, doi: 10.1007/s10762-016-0321-6.

[70] N. Kishimoto, S. Suzuki, A. Teranishi, and M. Asada, "Frequency Increase of Resonant Tunneling Diode Oscillators in Sub- $\mathrm{THz}$ and $\mathrm{THz}$ Range Using Thick Spacer Layers," Applied Physics Express, vol. 1, no. 4, pp. 042003, 2008, doi: 10.1143/apex.1.042003.

[71] H. Kanaya, R. Sogabe, T. Maekawa, S. Suzuki, and M. Asada, "Fundamental Oscillation up to $1.42 \mathrm{THz}$ in Resonant Tunneling Diodes by Optimized Collector Spacer Thickness," Journal of Infrared, Millimeter, and Terahertz Waves, vol. 35, no. 5, pp. 425-431, 2014, doi: 10.1007/s10762014-0058-z.

[72] S. Javalagi, V. Reddy, K. Gullapalli, and D. Neikirk, "High efficiency microwave diode oscillators," Electronics Letters, vol. 28, no. 18, pp. 1699-1701, 1992, doi: 10.1049/el:19921080.

[73] H. Kanaya, T. Maekawa, S. Suzuki, and M. Asada, "Structure dependence of oscillation characteristics of resonant-tunneling-diode terahertz oscillators associated with intrinsic and extrinsic delay times," Japanese
Journal of Applied Physics, vol. 54, no. 9, pp. 094103, 2015, doi: 10.7567/jjap.54.094103.

[74] L. V. Iogansen, "Resonance Tunneling of Electrons in Crystals," Journal of Experimental and Theoretical Physics, vol. 20, no. 1, pp. 180-185, 1965.

[75] E. O. Kane, "Basic Concepts of Tunneling," in Tunneling Phenomena in Solids, pp. 1-11, Springer, 1969.

[76] D. Bohm, "Quantum Theory," Dover Publications, 1989.

[77] J. H. Davies, "The Physics of Low-Dimensional Semiconductors," Cambridge University Press, 1998, doi: 10.1049/el:19921080.

[78] L. V. Iogansen, "The Possibility of Resonance Transmission of Electrons in Crystals Through a System of Barriers," Journal of Experimental and Theoretical Physics, vol. 18, no. 1, pp. 146-150, 1964.

[79] L. L. Chang, E. E. Mendez, and C. Tejedor, "Resonant Tunneling in Semiconductors: Physics and Applications," Springer, 1991.

[80] W. R. Frensley, "Quantum transport calculation of the small-signal response of a resonant tunneling diode," Applied Physics Letters, vol. 51, no. 6, pp. 448-450, 1987, doi: 10.1063/1.98418.

[81] T. C. L. G. Sollner, W. D. Goodhue, P. E. Tannenwald, C. D. Parker, and D. D. Peck, "Resonant tunneling through quantum wells at frequencies up to $2.5 \mathrm{THz}, "$ Applied Physics Letters, vol. 43, no. 6, pp. 588-590, 1983, doi: 10.1063/1.94434.

[82] J. S. Scott, J. P. Kaminski, M. Wanke, S. J. Allen, D. H. Chow, M. Lui, and T. Y. Liu, "Terahertz frequency response of an $\operatorname{In}_{0.53} \mathrm{Ga}_{0.47} \mathrm{As} / \mathrm{AlAs}$ resonant-tunneling diode," Applied Physics Letters, vol. 64, no. 15, pp. 1995-1997, 1994, doi: 10.1063/1.111717.

[83] A. Al-Khalidi, K. H. Alharbi, J. Wang, R. Morariu, L. Wang, A Khalid, J. M. L. Figueiredo, and E. Wasige, "Resonant Tunneling Diode Terahertz Sources With up to $1 \mathrm{~mW}$ Output Power in the J-Band," IEEE Transactions on Terahertz Science and Technology, vol. 10, no. 2, pp. 150-157, 2020, doi: 10.1109/TTHZ.2019.2959210.

[84] T. Maekawa, H. Kanaya, S. Suzuki, and M. Asada, "Oscillation up to $1.92 \mathrm{THz}$ in resonant tunneling diode by reduced conduction loss," Applied Physics Express, vol. 9, no. 2, pp. 024101, 2016, doi: 10.7567/apex.9.024101.

[85] S. Suzuki, M. Asada, A. Teranishi, H. Sugiyama, and H. Yokoyama, "Fundamental oscillation of resonant tunneling diodes above $1 \mathrm{THz}$ at room temperature," Applied Physics Letters, vol. 97, no. 24, pp. 242102, 2010, doi: $10.1063 / 1.3525834$

[86] H. Kanaya, H. Shibayama, R. Sogabe, S. Suzuki, and M. Asada, "Fundamental Oscillation up to $1.31 \mathrm{THz}$ in Resonant Tunneling Diodes with Thin Well and Barriers," Applied Physics Express, vol. 5, no. 12, pp. 124101, 2012, doi: 10.1143/apex.5.124101.

[87] S. Suzuki, A. Teranishi, K. Hinata, M. Asada, H. Sugiyama, and H. Yokoyama, "Fundamental Oscillation of up to $831 \mathrm{GHz}$ in GaInAs/AlAs Resonant Tunneling Diode," Applied Physics Express, vol. 2, pp. 054501, 2009, doi: 10.1143/apex.2.054501.

[88] R. Tsu and L. Esaki, "Tunneling in a finite superlattice," Applied Physics Letters, vol. 22, no. 11, pp. 562-564, 1973, doi: 10.1063/1.1654509.

[89] E. R. Brown, "High-Speed Resonant-Tunneling Diodes," in VLSI Electronics Microstructure Science, chapter 10, vol. 24, pp. 305-350, 1994 , doi: 10.1016/B978-0-12-234124-3.50015-X

[90] J. P. Sun, G. I. Haddad, P. Mazumder, and J. N. Schulman, ”Resonant tunneling diodes: models and properties," Proceedings of the IEEE, vol. 86, no. 4, pp. 641-660, 1998, doi: 10.1109/5.663541.

[91] J. N. Schulman, H. J. De Los Santos, and D. H. Chow, "Physics-based RTD current-voltage equation," IEEE Electron Device Letters, vol. 17, no. 5, pp. 220-222, 1996, doi: 10.1109/55.491835.

[92] J. Wang, A. Al-Khalidi, L. Wang, R. Morariu, A. Ofiare, and E. Wasige, "15-Gb/s 50-cm Wireless Link Using a High-Power Compact III-V 84-GHz Transmitter," IEEE Transactions on Microwave Theory and Techniques, vol. 66, no. 11, pp. 4698-4705, 2018, doi: 10.1109/TMTT.2018.2859983.

[93] M. Egard, M. Arlelid, L. Ohlsson, B. M. Borg, E. Lind, and L. -E. Wernersson, "In ${ }_{0.53} \mathrm{Ga}_{0.47}$ As RTD-MOSFET Millimeter-Wave Wavelet Generator," IEEE Electron Device Letters, vol. 33, no. 7, pp. 970-972, 2012, doi: 10.1109/LED.2012.2194132.

[94] L. Wang, "Reliable design of tunnel diode and resonant tunnelling diode based microwave sources," Ph.D. thesis, University of Glasgow, 2012.

[95] Q. Liu, A. Seabaugh, P. Chahal, and F. J. Morris, "Unified AC model for the resonant tunneling diode," IEEE Transactions on Electron Devices, vol. 51, no. 5, pp. 653-657, 2004, doi: 10.1109/TED.2004.825795.

[96] R. Lake and J. Yang, "A physics based model for the RTD quantum capacitance," IEEE Transactions on Electron Devices, vol. 50, no. 3, pp. 785-789, 2003, doi: 10.1109/TED.2003.811390. 
[97] T. P. E. Broekaert, B. Brar, J. P. A. van der Wagt, A. C. Seabaugh, F. J. Morris, T. S. Moise, E. A. Beam, and G. A. Frazier, "A monolithic 4-bit 2-Gsps resonant tunneling analog-to-digital converter,' IEEE Journal of Solid-State Circuits, vol. 33, no. 9, pp. 1342-1349, 1998, doi: 10.1109/4.711333.

[98] E. R. Brown, C. D. Parker, and T. C. L. G. Sollner, "Effect of quasibound-state lifetime on the oscillation power of resonant tunneling diodes," Applied Physics Letters, vol. 54, no. 10, pp. 934-936, 1989, doi: 10.1063/1.100812.

[99] W. F. Chow, "Principles of tunnel diode circuits," Wiley, 1964.

[100] M. N. Feiginov, "Does the quasibound-state lifetime restrict the highfrequency operation of resonant-tunnelling diodes?," Nanotechnology, vol. 11, no. 4, pp. 359-364, 2000, doi: 10.1088/0957-4484/11/4/333.

[101] E. R. Brown, W. D. Goodhue, and T. C. L. G. Sollner, 'Fundamental oscillations up to $200 \mathrm{GHz}$ in resonant tunneling diodes and new estimates of their maximum oscillation frequency from stationary-state tunneling theory," Journal of Applied Physics, vol. 64, no. 3, pp. 1519-1529, 1988, doi: $10.1063 / 1.341827$

[102] M. Asada, S. Suzuki, and N. Kishimoto, "Resonant Tunneling Diodes for Sub-Terahertz and Terahertz Oscillators," Japanese Journal of Applied Physics, vol. 47, no. 6, pp. 4375-4384, 2008, doi: 10.1143/jjap.47.4375.

[103] M. N. Feiginov, 'Displacement currents and the real part of highfrequency conductance of the resonant-tunneling diode," Applied Physics Letters, vol. 78, no. 21, pp. 3301-3303, 2001, doi: 10.1063/1.1372357.

[104] M. N. Feiginov, "Effect of the Coulomb interaction on the response time and impedance of the resonant-tunneling diodes," Applied Physics Letters, vol. 76, no. 20, pp. 2904-2906, 2000, doi: 10.1063/1.126512.

[105] R. Morariu, J. Wang, A. C. Cornescu, A. Al-Khalidi, A. Ofiare, J. M. L. Figueiredo, and E. Wasige, "Accurate Small-Signal Equivalent Circuit Modeling of Resonant Tunneling Diodes to $110 \mathrm{GHz}$," IEEE Transactions on Microwave Theory and Techniques, vol. 67, no. 11, pp. 4332-4340, 2019, doi: 10.1109/TMTT.2019.2939321.

[106] D. Cimbri, N. Weimann, Q. R. A. Al-Taai, A. Ofiare, and E. Wasige, "Ohmic Contacts Optimisation for High-Power InGaAs/AlAs DoubleBarrier Resonant Tunnelling Diodes Based on a Dual-Exposure EBeam Lithography Approach," International Journal of Nanoelectronics and Materials, 3rd International Conference on Applied Photonics and Electronics (InCAPE), 2021 (accepted).

[107] T. Wei, S. Stapleton, and O. Berolo, "Scattering parameter measurements of resonant tunneling diodes up to $40 \mathrm{GHz}$," IEEE Transactions on Electron Devices, vol. 42, no. 7, pp. 1378-1380, 1995, doi: $10.1109 / 16.391225$

[108] C. Kidner, I. Mehdi, J. R. East, and G. I. Haddad, "Bias circuit instabilities and their effect on the d.c. current-voltage characteristics of double-barrier resonant tunneling diodes," Solid-State Electronics, vol. 34, no. 2, pp. 149-156, 1991, doi: 10.1016/0038-1101(91)90081-9.

[109] L. Wang, J. M. L. Figueiredo, C. N. Ironside, and E. Wasige, "DC Characterization of Tunnel Diodes Under Stable Non-Oscillatory Circuit Conditions," IEEE Transactions on Electron Devices, vol. 58, no. 2, pp. 343-347, 2011, doi: 10.1109/TED.2010.2091507.

[110] M. Feiginov, C. Sydlo, O. Cojocari, and P. Meissner, "High-frequency nonlinear characteristics of resonant-tunnelling diodes," Applied Physics Letters, vol. 99, no. 13, pp. 133501, 2011, doi: 10.1063/1.3644491.

[111] S. Diebold, S. Nakai, K. Nishio, J. Kim, K. Tsuruda, T. Mukai, M. Fujita, and T. Nagatsuma, "Modeling and Simulation of Terahertz Resonant Tunneling Diode-Based Circuits," IEEE Transactions on Terahertz Science and Technology, vol. 6, no. 5, pp. 716-723, 2016, doi: 10.1109/TTHZ.2016.2592180.

[112] S. Clochiatti, K. Aikawa, K. Arzi, E. Mutlu, M. Suhara, N. Weimann, and W. Prost, "Large-Signal Modelling of sub-THz InP Triple-Barrier Resonant Tunneling Diodes," Third International Workshop on Mobile Terahertz Systems (IWMTS), pp. 1-5, 2020, doi: 10.1109/IWMTS49292.2020.9166270.

[113] E. R. Brown, C. D. Parker, S. Verghese, M. W. Geis, and J F. Harvey, "Resonant-tunneling transmission-line relaxation oscillator," Applied Physics Letters, vol. 70, no. 21, pp. 2787-2789, 1997, doi: 10.1063/1.119059.

[114] C. L. Chen, R. H. Mathews, L. J. Mahoney, S. D. Calawa, J. P. Sage, K M. Molvar, C. D. Parker, P. A. Maki, and T. C. L. G. Sollner, ”Resonanttunneling-diode relaxation oscillator," Solid-State Electronics, vol. 44, no. 10, pp. 1853-1856, 2000, doi: 10.1016/S0038-1101(00)00105-2.

[115] M. E. Hines, "High-frequency negative-resistance circuit principles for Esaki diode applications," The Bell System Technical Journal, vol. 39, no. 3, pp. 477-513, 1960, doi: 10.1002/j.1538-7305.1960.tb03933.x.

[116] C. Kidner, I. Mehdi, J. R. East, and G. I. Haddad, "Power and stability limitations of resonant tunneling diodes," IEEE Transactions on
Microwave Theory and Techniques, vol. 38, no. 7, pp. 864-872, 1990, doi: $10.1109 / 22.55778$.

[117] L. Wang, "Output Power Analysis and Simulations of Resonant Tunneling Diode Based Oscillators," International Computer Science Conference (ICSC): System Simulation and Scientific Computing, pp. 47-55, 2012, doi: 10.1007/978-3-642-34381-0_6.

[118] J. Wang, A. Al-Khalidi, K. Alharbi, A. Ofiare, H. Zhou, E. Wasige, and J. Figueiredo, "High performance resonant tunneling diode oscillators as terahertz sources," 46th European Microwave Conference (EuMC), pp. 341-344, 2016, doi: 10.1109/EuMC.2016.7824348.

[119] S. Diebold, K. Nishio, Y. Nishida, J. -Y. Kim, K. Tsuruda, T. Mukai, M. Fujita, and T. Nagatsuma, "High-speed error-free wireless data transmission using a terahertz resonant tunnelling diode transmitter and receiver,' Electronics Letters, vol. 52, no. 24, pp. 1999-2001, 2016, doi: 10.1049/el.2016.2941.

[120] E. Wasige, A. Al-Khalidi, K. Alharbi, and J. Wang, "High performance microstrip resonant tunneling diode oscillators as terahertz sources," 9th UK-Europe-China Workshop on Millimetre Waves and Terahertz Technologies (UCMMT), pp. 25-28, 2016, doi: 10.1109/UCMMT.2016.7873950.

[121] S. Suzuki, M. Shiraishi, H. Shibayama, and M. Asada, "High-Power Operation of Terahertz Oscillators With Resonant Tunneling Diodes Using Impedance-Matched Antennas and Array Configuration," IEEE Journal of Selected Topics in Quantum Electronics, vol. 19, no. 1, pp. 8500108-8500108, 2013, doi: 10.1109/JSTQE.2012.2215017.

[122] M. Reddy, "Schottky-collector Resonant Tunnel Diodes for SubMillimeter-Wave Applications," Ph.D. thesis, University of California Santa Barbara, 1997.

[123] D. Horikawa, Y. Chen, T. Koike, S. Suzuki, and M. Asada, "Resonant tunneling-diode terahertz oscillator integrated with a radial line slot antenna for circularly polarized wave radiation," Semiconductor Science and Technology, vol. 33, no. 11, pp. 114005, 2018, doi: 10.1088/13616641/aae1ef.

[124] K. Okada, K. Kasagi, N. Oshima, S. Suzuki, and M. Asada, ”ResonantTunneling-Diode Terahertz Oscillator Using Patch Antenna Integrated on Slot Resonator for Power Radiation," IEEE Transactions on Terahertz Science and Technology, vol. 5, no. 4, pp. 613-618, 2015, doi: 10.1109/TTHZ.2015.2441740.

[125] K. Urayama, S. Aoki, S. Suzuki, M. Asada, H. Sugiyama, and H. Yokoyama, "Sub-Terahertz Resonant Tunneling Diode Oscillators Integrated with Tapered Slot Antennas for Horizontal Radiation," Applied Physics Express, vol. 2, no. 4, pp. 044501, 2019, doi: 10.1143/apex.2.044501.

[126] J. Lee, M. Kim, and K. Yang, "A 1.52 THz RTD Triple-Push Oscillator With a $\mu \mathrm{W}$-Level Output Power,' IEEE Transactions on Terahertz Science and Technology, vol. 6, no. 2, pp. 336-340, 2016, doi: 10.1109/TTHZ.2015.2509358.

[127] K. Kasagi, S. Suzuki, and M. Asada, "Large-scale array of resonant tunneling- diode terahertz oscillators for high output power at $1 \mathrm{THz}$,' Journal of Applied Physics, vol. 125, no. 15, pp. 151601, 2009, doi: 10.1063/1.5051007.

[128] M. Kim, J. Lee, J. Lee, and K. Yang, "A 675 GHz Differential Oscillator Based on a Resonant Tunneling Diode," IEEE Transactions on Terahertz Science and Technology, vol. 6, no. 3, pp. 510-512, 2016 , doi: 10.1109/TTHZ.2016.2554399.

[129] K. Kasagi, S. Fukuma, S. Suzuki, and M. Asada, "Proposal and fabrication of dipole array antenna structure in resonant-tunneling-diode terahertz oscillator array," 41st International Conference on Infrared, Millimeter, and Terahertz waves (IRMMW-THz), pp. 1-2, 2016, doi: 10.1109/IRMMW-THz.2016.7758957.

[130] K. H. Alharbi, A. Khalid, A. Ofiare, J. Wang, and E. Wasige, ”Diced and grounded broadband bow-tie antenna with tuning stub for resonant tunnelling diode terahertz oscillators," IET Colloquium on MillimetreWave and Terahertz Engineering \& Technology 2016, pp. 1-4, 2016, doi: 10.1049/ic.2016.0013.

[131] M. Reddy, S. C. Martin, A. C. Molnar, R. E. Muller, R. P. Smith, P. H. Siegel, M. J. Mondry, M. J. W. Rodwell, H. Kroemer, and S. J. Allen, "Monolithic Schottky-collector resonant tunnel diode oscillator arrays to 650 GHz," IEEE Electron Device Letters, vol. 18, no. 5, pp. 218-221, 1997, doi: 10.1109/55.568771.

[132] N. Orihashi, S. Suzuki, and M. Asada, "One THz harmonic oscillation of resonant tunneling diodes," Applied Physics Letters, vol. 87, no. 23, pp. 233501, 2005, doi: 10.1063/1.2139850.

[133] R. E. Neidert, S. C. Binari, and T. Weng, 'Dielectric constant of semiinsulating indium phosphide," Electronics Letters, vol. 18, no. 23, pp. 987-988, 1982, doi: 10.1049/el:19820675. 
[134] C. Hilsum, S. Fray, and C. Smith, "The optical frequencies and dielectric constants of InP," Solid State Communications, vol. 7, no. 15, pp. 1057-1059, 1969, doi: 10.1016/0038-1098(69)90470-0.

[135] K. Hinata, M. Shiraishi, S. Suzuki, M. Asada, H. Sugiyama, and H. Yokoyama, "Sub-Terahertz Resonant Tunneling Diode Oscillators with High Output Power $(\sim 200 \mu \mathrm{W})$ Using Offset-Fed Slot Antenna and High Current Density," Applied Physics Express, vol. 3, no. 1, pp. 014001, 2009, doi: 10.1143/apex.3.014001.

[136] K. Hinata, M. Shiraishi, S. Suzuki, M. Asada, H. Sugiyama, and H. Yokoyama, "High Output Power $(\sim 400 \mu \mathrm{W})$ Oscillators at around 550 GHz Using Resonant Tunneling Diodes with Graded Emitter and Thin Barriers," Applied Physics Express, vol. 4, no. 6, pp. 064101, 2011, doi: 10.1143/apex.4.064101.

[137] R. Izumi, T. Sato, S. Suzuki, and M. Asada, "Resonant-tunnelingdiode terahertz oscillator with a cylindrical cavity for high-frequency oscillation," AIP Advances, vol. 9, no. 8, pp. 085020, 2019, doi: $10.1063 / 1.5114963$.

[138] F. Han, K. Kobayashi, S. Suzuki, H. Tanaka, H. Fujikata, and M. Asada, "Impedance matching in high-power resonant-tunneling-diode terahertz oscillators integrated with rectangular-cavity resonator," IEICE Transactions on Electronics, vol. E104.C, no. 8, pp. 398-402, 2021, doi: 10.1587/transele.2020ECS6020.

[139] X. Yu, Y. Suzuki, M. Van Ta, S. Suzuki, and M. Asada, "Highly Efficient Resonant Tunneling Diode Terahertz Oscillator With a Split Ring Resonator," IEEE Electron Device Letters, vol. 42, no. 7, pp. 982-985, 2021, doi: 10.1109/LED.2021.3082577.

[140] T. V. Mai, Y. Suzuki, X. Yu, S. Suzuki, and M. Asada, "Structure-Simplified Resonant-Tunneling-Diode Terahertz Oscillator Without Metal-Insulator-Metal Capacitors," Journal of Infrared, Millimeter, and Terahertz Waves, vol. 41, no. 12, pp. 1498-1507, 2020, doi: 10.1007/s10762-020-00738-7.

[141] S. Kitagawa, S. Suzuki, and M. Asada, "Wide frequencytunable resonant tunnelling diode terahertz oscillators using varactor diodes," Electronics Letters, vol. 52, no. 6, pp. 479-481, 2016, doi: 10.1049/el.2015.3921.

[142] J. Lee, M. Kim, J. Lee and K. Yang, "A sub-mW D-band $2^{\text {nd }}$ harmonic oscillator using InP-based quantum-effect tunneling devices," 26th International Conference on Indium Phosphide and Related Materials (IPRM), pp. 1-2, 2014, doi: 10.1109/ICIPRM.2014.6880527.

[143] N. Nishigami, Y. Nishida, S. Diebold, J. Kim, M. Fujita. and T. Nagatsuma, "Resonant Tunneling Diode Receiver for Coherent Terahertz Wireless Communication," 2018 Asia-Pacific Microwave Conference (APMC), pp. 726-728, 2018, doi: 10.23919/APMC.2018.8617565.

[144] Y. Takida, S. Suzuki, M. Asada, and H. Minamide, "Sensitive terahertzwave detector responses originated by negative differential conductance of resonant-tunneling-diode oscillator," Applied Physics Letters, vol. 117, no. 2, pp. 021107 , 2020, doi: 10.1063/5.0012318.

[145] D. Pozar, "Microwave and RF Design of Wireless Systems," Wiley, 2000

[146] T. Shiode, T. Mukai, M. Kawamura, and T. Nagatsuma, "Giga-bit wireless communication at $300 \mathrm{GHz}$ using resonant tunneling diode detector," Asia-Pacific Microwave Conference 2011, pp. 1122-1125, 2011.

[147] D. Schoenherr, C. Bleasdale, T. Goebel, C. Sydlo, H. L. Hartnagel, R. Lewis, and P. Meissner, "Extremely broadband characterization of a Schottky diode based $\mathrm{THz}$ detector," 35th International Conference on Infrared, Millimeter, and Terahertz Waves, pp. 1-2, 2010, doi: 10.1109/ICIMW.2010.5613008.

[148] S. Boppel, A. Lisauskas, M. Mundt, D. Seliuta, L. Minkevicius, I Kasalynas, G. Valusis, M. Mittendorff, S. Winnerl, V. Krozer, and H. G. Roskos, "CMOS Integrated Antenna-Coupled Field-Effect Transistors for the Detection of Radiation From 0.2 to $4.3 \mathrm{THz}, "$ IEEE Transactions on Microwave Theory and Techniques, vol. 60, no. 12, pp. 3834-3843, 2012, doi: 10.1109/TMTT.2012.2221732.

[149] M. Bauer, A. Rämer, S. A. Chevtchenko, K. Y. Osipov, D. Čibiraitè, S Pralgauskaite, K. Ikamas, A. Lisauskas, W. Heinrich, V. Krozer, and H. G. Roskos, "A High-Sensitivity AlGaN/GaN HEMT Terahertz Detector With Integrated Broadband Bow-Tie Antenna," IEEE Transactions on Terahertz Science and Technology, vol. 9, no. 4, pp. 430-444, 2019, doi: 10.1109/TTHZ.2019.2917782.

[150] S. -P. Han, H. Ko, J. -W. Park, N. Kim, Y. -J. Yoon, J. -H. Shin, D. Y. Kim, D. H. Lee, and K. H. Park, "InGaAs Schottky barrier diode array detector for a real-time compact terahertz line scanner," Optics Express, vol. 21, no. 22, pp. 25874-25882, 2013, doi: 10.1364/OE.21.025874.

[151] X. Yu, J. -Y. Kim, M. Fujita, and T. Nagatsuma, "Efficient mode converter to deep-subwavelength region with photonic-crystal waveguide platform for terahertz applications," Optics Express, vol. 27, no. 20, pp. 28707-28721, 2019, doi: 10.1364/OE.27.028707.
[152] Y. Takida, S. Suzuki, M. Asada, and H. Minamide, "Sensitivity Measurement of Resonant-Tunneling-Diode Terahertz Detectors," 44th International Conference on Infrared, Millimeter, and Terahertz Waves (IRMMW-THz), pp. 1-2, 2013, doi: 10.1109/IRMMW-THz.2019.8873774

[153] Y. Takida, S. Suzuki, M. Asada, and H. Minamide, "Sensitive Continuous-Wave Terahertz Detection by Resonant-Tunneling-Diode Oscillators," 45th International Conference on Infrared, Millimeter, and Terahertz Waves (IRMMW-THz), pp. 1-2, 2020, doi: 10.1109/IRMMWTHz46771.2020.9370758.

[154] R. Morariu, "Accurate Characterisation of Resonant Tunnelling Diodes for High-Frequency Applications," Ph.D. thesis, University of Glasgow, 2021.

[155] K. Arzi, S. Clochiatti, E. Mutlu, A. Kowaljow, B. Sievert, D. Erni, N. Weimann, and W. Prost, "Broadband Detection capability of a Triple Barrier Resonant Tunneling Diode," 2nd International Workshop on Mobile Terahertz Systems (IWMTS), pp. 1-4, 2019, doi: 10.1109/IWMTS.2019.8823724.

[156] K. Arzi, S. Clochiatti, S. Suzuki, A. Rennings, D. Erni, N. Weimann, M. Asada, and W. Prost, "Triple-Barrier Resonant-Tunnelling Diode THz Detectors with on-chip antenna," 12th German Microwave Conference (GeMiC), pp. 17-19, 2019, doi: 10.23919/GEMIC.2019.8698124

[157] Y. Koyama, R. Sekiguchi, and T. Ouchi, "Oscillations up to $1.40 \mathrm{THz}$ from Resonant-Tunneling-Diode-Based Oscillators with Integrated Patch Antennas," Applied Physics Express, vol. 6, no. 6, pp. 064102, 2013, doi: 10.7567/apex.6.064102.

[158] R. Sekiguchi, Y. Koyama, and T. Ouchi, "Subterahertz oscillations from triple-barrier resonant tunneling diodes with integrated patch antennas," Applied Physics Letters, vol. 96, no. 6, pp. 062115, 2010, doi: $10.1063 / 1.3315868$.

[159] K. Arzi, A. Rennings, D. Erni, N. Weimann, W. Prost, S. Suzuki, and M. Asada, "Millimeter-wave Signal Generation and Detection via the same Triple Barrier RTD and on-chip Antenna," 1st International Workshop on Mobile Terahertz Systems (IWMTS), pp. 1-4, 2018, doi: 10.1109/IWMTS.2018.8454700.

[160] F. Sizov, "Terahertz radiation detectors: the state-of-the-art," Semiconductor Science and Technology, vol. 33, no. 12, pp. 123001, 2018, doi: 10.1088/1361-6641/aae473.

[161] M. Asada and S. Suzuki, "Theoretical analysis of coupled oscillator array using resonant tunneling diodes in subterahertz and terahertz range," Journal of Applied Physics, vol. 103, no. 12, pp. 124514, 2008, doi: $10.1063 / 1.2947600$

[162] K. Arzi, S. Suzuki, A. Rennings, D. Erni, N. Weimann, M. Asada, and W. Prost, "Subharmonic Injection Locking for Phase and Frequency Control of RTD-Based THz Oscillator," IEEE Transactions on Terahertz Science and Technology, vol. 10, no. 2, pp. 221-224, 2020, doi: 10.1109/TTHZ.2019.2959411.

[163] Y. Nishida, N. Nishigami, S. Diebold, J. Kim, M. Fujita, and T. Nagatsuma, "Terahertz coherent receiver using a single resonant tunnelling diode," Scientific Reports, vol. 9, no. 1, pp. 18125, 2019, doi: 10.1038/s41598-019-54627-8.

[164] A. Al-Khalidi, J. Wang, and E. Wasige, "Compact J-band Oscillators With $1 \mathrm{~mW}$ RF output Power and Over $110 \mathrm{GHz}$ Modulation Bandwidth," 43rd International Conference on Infrared, Millimeter, and Terahertz Waves (IRMMW-THz), pp. 1-2, 2018, doi: 10.1109/IRMMWTHz.2018.8510099.

[165] J. Wang, A. Al-Khalidi, S. Ahearne, and E. Wasige, "1080P HD Video Transmission using RTD Transmitter," 14th UK-Europe-China Workshop on Millimetre-Waves and Terahertz Technologies (UCMMT), pp. 1-3, 2021, doi: 10.1109/UCMMT53364.2021.9569915.

[166] L. Ohlsson and L. Wernersson, "A 15-Gb/s Wireless ON-OFF Keying Link," IEEE Access, vol. 2, pp. 1307-1313, 2014, doi: 10.1109/ACCESS.2014.2364638.

[167] N. Oshima, K. Hashimoto, S. Suzuki, and M. Asada, "Wireless data transmission of $34 \mathrm{Gbit} / \mathrm{s}$ at a $500-\mathrm{GHz}$ range using resonant-tunnellingdiode terahertz oscillator," Electronics Letters, vol. 52, no. 22, pp. 1897 1898, 2016, doi: 10.1049/el.2016.3120.

[168] M. Asada and S. Suzuki, "Terahertz Oscillators Using Resonant Tunneling Diodes," Asia-Pacific Microwave Conference (APMC), pp. 521523, 2018, doi: 10.23919/APMC.2018.8617175

[169] K. Nishio, S. Diebold, S. Nakai, K. Tsuruda, T. Mukai, J. -Y. Kim, M. Fujita, and T. Nagatsuma, "Resonant Tunneling Diode Receivers for 300-GHz-band Wireless Communications," URSI-Japan Radio Science Meeting, 2015.

[170] N. Oshima, K. Hashimoto, S. Suzuki, and M. Asada, "Terahertz Wireless Data Transmission With Frequency and Polarization Division Multiplexing Using Resonant-Tunneling-Diode Oscillators," IEEE Trans- 
actions on Terahertz Science and Technology, vol. 7, no. 5, pp. 593-598, 2017, doi: 10.1109/TTHZ.2017.2720470.

[171] X. Yu, R. Yamada, J. Kim, M. Fujita, and T. Nagatsuma, "Integrated Circuits Using Photonic-Crystal Slab Waveguides and Resonant Tunneling Diodes for Terahertz Communication," Progress in Electromagnetics Research Symposium (PIERS-Toyama), pp. 599-605, 2018, doi: 10.23919/PIERS.2018.8597616.

[172] T. Yamamoto, N. Nishigami, Y. Nishida, M. Fujita, and T. Nagatsuma, "16-QAM Wireless Communications Using Resonant Tunneling Diodes at $60 \mathrm{Gbit} / \mathrm{s,"} \mathrm{IEICE} \mathrm{General} \mathrm{Conference,} 2020$.

[173] J. Webber, A. Oshiro, S. Iwamatsu, Y. Nishida, M. Fujita, and T. Nagatsuma, "48-Gbit/s 8K video-transmission using resonant tunnelling diodes in 300-GHz band," Electronics Letters, vol. 57, no. 17, pp. 668669, 2021, doi: 10.1049/ell2.12219.

[174] S. Iwamatsu, N. Nishigami, Y. Nishida, M. Fujita, and T. Nagatsuma, "Broadband terahertz resonant tunnelling diode transmitter integrated with coplanar-waveguide-fed slot-ring antenna," Electronics Letters, vol. 57, no. 25, pp. 1001-1003, 2021, doi: 10.1049/ell2.12332.

[175] J. Webber, N. Nishigami, X. Yu, J. Kim, M. Fujita, and T. Nagatsuma, "Terahertz Wireless Communication using Resonant Tunneling Diodes and Practical Radio-over-Fiber Technology," IEEE International Conference on Communications Workshops (ICC Workshops), pp. 1-5, 2019, doi: 10.1109/ICCW.2019.8757176.

[176] J. Webber, N. Nishigami, J. Kim, M. Fujita, and T. Nagatsuma, "Terahertz Wireless CDMA Communication Using Resonant Tunneling Diodes," IEEE Globecom Workshops (GC Wkshps), pp. 1-6, 2019, doi: 10.1109/GCWkshps45667.2019.9024595.

[177] E. R. Brown, C. D. Parker, A. R. Calawa, M. J. Manfra, and K. M. Molvar, "A quasioptical resonant-tunneling-diode oscillator operating above $200 \mathrm{GHz}$," IEEE Transactions on Microwave Theory and Techniques, vol. 41, no. 4, pp. 720-722, 1993, doi: 10.1109/22.231671.

[178] T. Fujii, H. Mazaki, F. Takei, J. Bae, M. Narihiro, T. Noda, H. Sakaki, and K. Mizuno, "Coherent power combining of millimeter wave resonant tunneling diodes in a quasi-optical resonator," IEEE MTT-S International Microwave Symposium Digest, vol. 2, pp. 919-922, 1993, doi: 10.1109/MWSYM.1996.511177.

[179] E. Ozbay, D. M. Bloom, D. H. Chow and, J. N. Schulman, "1.7-ps, microwave, integrated-circuit-compatible InAs/AlSb resonant tunneling diodes," IEEE Electron Device Letters, vol. 14, no. 8, pp. 400-402, 1993, doi: $10.1109 / 55.225592$.

[180] J. R. Söderström, E. R. Brown, C. D. Parker, L. J. Mahoney, J. Y. Yao, T. G. Andersson, and T. C. McGill, "Growth and characterization of high current density, high-speed InAs/AlSb resonant tunneling diodes," Applied Physics Letters, vol. 58, no. 3, pp. 275-277, 1991, doi: 10.1063/1.104659.

[181] E. R. Brown, S. J. Eglash, G. W. Turner, C. D. Parker, J. V. Pantano, and D. R. Calawa, "Effect of lattice-mismatched growth on InAs/AlSb resonant-tunneling diodes," IEEE Transactions on Electron Devices, vol. 41, no. 6, pp. 879-882, 1994, doi: 10.1109/16.293296.

[182] E. R. Brown and C. D. Parker, "Resonant tunnel diodes as submillimetre-wave sources," Philosophical Transactions of the Royal Society of London. Series A: Mathematical, Physical and Engineering Sciences, vol. 354, no. 1717, pp. 2365-2381, 1996, doi: 10.1098/rsta.1996.0105.

[183] T. Miyamoto, A. Yamaguchi, and T. Mukai, "Terahertz imaging system with resonant tunneling diodes," Japanese Journal of Applied Physics, vol. 55, no. 3, pp. 032201, 2016, doi: 10.7567/jjap.55.032201.

[184] K. Okamoto, K. Tsuruda, S. Diebold, S. Hisatake, M. Fujita, and T. Nagatsuma, "Terahertz Sensor Using Photonic Crystal Cavity and Resonant Tunneling Diodes," Journal of Infrared, Millimeter, and Terahertz Waves, pp. 1085-1087, 2017, doi: 10.1007/s10762-017-0391-0.

[185] L. Yi, R. Kaname, Y. Nishida, X. Yu, M. Fujita, and T. Nagatsuma, "Imaging Applications with a Single Resonant Tunneling Diode Transceiver in 300-GHz Band," International Topical Meeting on Microwave Photonics (MWP), pp. 120-123, 2020, doi: 10.23919/MWP48676.2020.9314482.

[186] S. Kitagawa, M. Mizuno, S. Saito, K. Ogino, S. Suzuki, and M. Asada, "Frequency-tunable resonant-tunneling-diode terahertz oscillators applied to absorbance measurement," Japanese Journal of Applied Physics, vol. 56, no. 5, pp. 058002, 2017, doi: 10.7567/jjap.56.058002.

[187] A. Dobroiu, R. Wakasugi, Y. Shirakawa, S. Suzuki, and M. Asada, "Amplitude-modulated continuous-wave radar in the terahertz range using lock-in phase measurement," Measurement Science and Technology, vol. 31, no. 10, pp. 105001, 2020, doi: 10.1088/1361-6501/ab912a.

[188] A. Dobroiu, Y. Shirakawa, S. Suzuki, M. Asada, and H. Ito, "Subcarrier Frequency-Modulated Continuous-Wave Radar in the Terahertz Range Based on a Resonant-Tunneling-Diode Oscillator," Sensors, vol. 20, no. 23, pp. 6848, 2020, doi: $10.3390 / \mathrm{s} 20236848$.
[189] H. Konno, A. Dobroiu, S. Suzuki, M. Asada, and H. Ito, ”Discrete Fourier Transform Radar in the Terahertz-Wave Range Based on a Resonant-Tunneling-Diode Oscillator," Sensors, vol. 21, no. 13, pp. 4367, 2020, doi: $10.3390 / \mathrm{s} 21134367$.

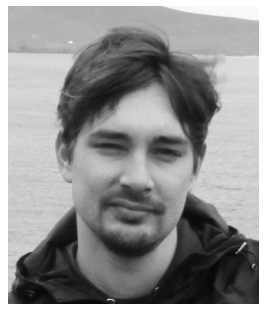

Davide Cimbri received the B.Sc. in Applied Physics and the M.Sc. in Nanotechnology (both cum laude) from Politecnico di Torino, Torino, Italy. He worked on the M.Sc. thesis at Boston University, Boston, Massachusetts, U.S., working on quantum-mechanical computational modelling of antimonides-based type-II superlattice mid-infrared (MIR) photo-detectors.

$\mathrm{He}$ is currently with the High-Frequency Electronics group, division of Electronics and Nanoscale Engineering, James Watt School of Engineering, University of Glasgow, Glasgow, United Kingdom, working towards the Ph.D. degree in Electronics and Electrical Engineering. His research focuses on resonant tunnelling diodes (RTDs) for terahertz (THz) applications.

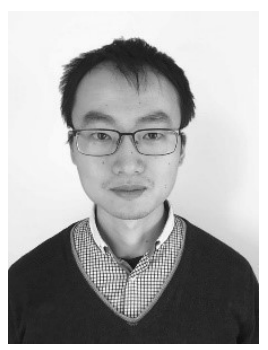

Jue Wang received the $\mathrm{PhD}$ degree in Electronics and Electrical Engineering from the University of Glasgow in 2014.

From 2014, he has been working on $\mathrm{THz}$ device technology including high power sources and high-sensitive detectors based on resonant tunneling diode. His research also focuses on terahertz applications including ultrafast wireless communications, $\mathrm{THz}$ imaging, etc.

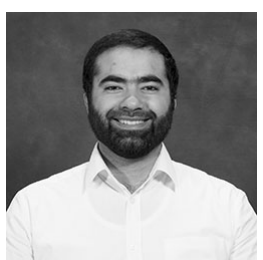

Abdullah Al-Khalidi received the BEng, MSc and $\mathrm{PhD}$ degrees from the University of Glasgow in 2010, 2011 and 2015, respectively.

$\mathrm{He}$ is currently a Lecturer at the University of Glasgow. From 2015-2019, he was a postdoctoral researcher at the University of Glasgow. His main research interests include $\mathrm{THz}$ resonant tunnelling diodes (RTDs) and gallium nitride $(\mathrm{GaN})$ transistor technologies.

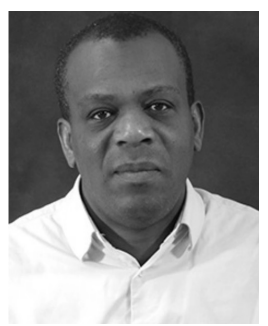

Edward Wasige received the B.Sc. (Eng.) degree in electrical engineering from the University of Nairobi, Nairobi, Kenya, in 1988, the M.Sc. (Eng.) degree in microelectronic systems and telecommunications from the University of Liverpool, Liverpool, U.K., in 1990, and the Dr.-Ing. degree in electrical engineering from Kassel University, Kassel, Germany, in 1999.

In 1990-93 and 1999-2001, he was a lecturer at Moi University in Kenya. In 2001-02, he was a UNESCO Postdoctoral Fellow with the Technion - Israel Institute of Technology. He has been a lecturer at the University of Glasgow since 2002, where he is now a professor in high frequency electronics. His research interests include compound semiconductor micro-/nanoelectronics and applications with focus on gallium nitride $(\mathrm{GaN})$ electronics and resonant tunnelling diode based terahertz electronics. 